\title{
Upper Semicontinuity of Attractors for Approximations of Semigroups and Partial Differential Equations
}

\author{
By Jack K. Hale, Xiao-Biao Lin, and Geneviève Raugel
}

\begin{abstract}
Suppose a given evolutionary equation has a compact attractor and the evolutionary equation is approximated by a finite-dimensional system. Conditions are given to ensure the approximate system has a compact attractor which converges to the original one as the approximation is refined. Applications are given to parabolic and hyperbolic partial differential equations.
\end{abstract}

1. Introduction. Suppose $X$ is a Banach space and $T(t), t \geq 0$, is a $C^{r}$. semigroup on $X$ with $r \geq 0$; that is, $T(t), t \geq 0$, is a semigroup with $T(t)$ continuous in $t, x$ together with the derivatives in $x$ up through the order $r$.

Following standard terminology (see, for instance, Hale [12]), a set $B \subset X$ is said to attract a set $C \subset X$ under the semigroup $T(t)$ if, for any $\varepsilon>0$, there is a $t_{0}=t_{0}(B, C, \varepsilon)$ such that $T(t) C \subset \mathcal{N}(B, \varepsilon)$ for $t \geq t_{0}$, where $\mathcal{N}(B, \varepsilon)$ denotes the $\varepsilon$-neighborhood of $B$. A compact invariant set $A$ is said to be a local attractor if there exists an open neighborhood $U$ of $A$ such that $A$ attracts $U$. The set $A$ is an attractor if, for any bounded set $B$ in $X, A$ attracts $B$. Conditions for the existence of an attractor may be found in Hale [12].

Now suppose the semigroup depends on a parameter $\lambda$ belonging to an open subset of a Banach space, say $T(t)=T_{\lambda}(t)$, where $T_{\lambda}(t) x$ is continuous in $(t, x, \lambda)$, the continuity in $\lambda$ being uniform on bounded sets. If $A_{\lambda_{0}}$ is a local attractor for $T_{\lambda_{0}}(t)$, then additional smoothing properties of $T_{\lambda}(t)$ will imply there is a neighborhood $V$ of $\lambda_{0}$ such that $T_{\lambda}(t), \lambda \in V$, has a local attractor $A_{\lambda}$ and $A_{\lambda}$ is upper semicontinuous at $\lambda_{0}$, that is, $\delta_{X}\left(A_{\lambda}, A_{\lambda_{0}}\right) \rightarrow 0$ as $\lambda \rightarrow \lambda_{0}$ where, for any two subsets $A, B$ of $X$,

$$
\delta_{X}(A, B)=\sup _{x \in A} \operatorname{dist}_{X}(x, B) \quad \text { and } \quad \operatorname{dist}_{X}(x, B)=\inf _{x \in B}\|x-y\|_{X} .
$$

The most general result of this type is due to Cooperman [7] and may be found also in Hale [11]. The result for gradient systems is in Hale [12].

The spirit of this paper relates to the above property of upper semicontinuity of a local attractor. Here we consider semigroups $T_{h}(t)$ depending on a parameter $h>0$ which "approximate" the semigroup $T(t)$ and give conditions under which there exists a local attractor $A_{h}$ for $T_{h}(t)$ with the property that $\delta_{X}\left(A_{h}, A\right) \rightarrow 0$ as $h \rightarrow 0$. The essential difference between the results here and the ones mentioned before

Received August 11, 1986.

1980 Mathematics Subject Classification (1985 Revision). Primary 35A40, 65N30.

*This research was supported by the Air Force Office of Scientific Research under Grant \#AFAFOSR 84-0376; by the U. S. Army Research Office under Grant \#DAAG-29-83-K-0029; and by the National Science Foundation under Grant \#DMS-8507056. 
is that the approximate semigroups can correspond to Galerkin approximations, splines or discretizations in time of evolutionary equations. These approximations have no uniform continuity property with respect to $h$.

The outline of the paper is as follows. In Section 2 we give a general approximation result which attempts to bring out the essential elements of the approximate and exact semigroups to ensure that there is a local, compact attractor which is upper semicontinuous. We also give one result in which we assume the approximate semigroups have a local compact attractor and then infer that the exact semigroup has a compact attractor. For the Navier-Stokes equation and the case in which the local attractor for each approximation is a point, Constantin, Foias and Temam [6] have given conditions which ensure that the original equations have an equilibrium. Schmitt, Thompson and Walter [31] discuss the solution of an elliptic boundary value problem in an infinite strip by analyzing solutions of approximate differential equations. This aspect of the problem is important but much more difficult and will be developed further in subsequent publications. The remainder of the paper is devoted to giving specific approximation schemes for particular evolutionary systems for which the hypotheses of Section 2 are satisfied. These applications include spectral projection methods for sectorial evolutionary equations and Galerkin approximations for parabolic equations as well as discretizations in time. Some results about the approximation of the Navier-Stokes equations and of a damped hyperbolic wave equation are also given.

In this paper the convergence of the attractor $A_{h}$ to $A$ as $h \rightarrow 0$ is considered only in the sense of sets. The relationship between the dynamics on the attractors also must be discussed. This problem is much more difficult and requires some knowledge of the flow on $A$. Some results on the case in which the flow on $A$ is Morse-Smale have already been obtained and will appear in Lin and Raugel [25]. For the case of a scalar parabolic equation in one space dimension with a cubic nonlinearity, this latter property has been discussed for space approximation using the Conley index (Khalsa [22]). Numerical computations using Galerkin approximations have been done for a similar example (Mora [28], Rutkowski [30]).

2. A General Approximation Result. In this section we give a general result on the approximation of a local attractor by "approximate" semigroups. These results are very similar to local versions of the ones of Cooperman [7] or Hale [11]. More precisely, let $h>0$ be a parameter which will tend to 0 and let $\left(X_{h}\right)_{h}$ be a family of subspaces of $X$ such that

$$
\lim _{h \rightarrow 0} \operatorname{dist}_{X}\left(x, X_{h}\right)=0 \quad \text { for any } x \text { in } X
$$

Let $T_{h}(t), t \geq 0$, be a $C^{s}$-semigroup on $X_{h}$ with $s \geq 0$. Actually, $T_{h}(t) x_{h}$ need not be a priori defined for all $t>0$. More precisely, we shall only assume that $T_{h}(0)=\operatorname{Id}_{X_{h}}, T_{h}(t+s) x_{h}=T_{h}(s) T_{h}(t) x_{h}$ for $s \geq 0, t \geq 0$ (as soon as $T_{h}(t+s) x_{h}$ and $T_{h}(s) T_{h}(t) x_{h}$ are well defined), that $T_{h}(t) x_{h}$ is continuous in $t$ and $x_{h}$ when it is defined and finally, that $T_{h}(t) x_{h}$ is left-continuous at $t_{1}$ if $T_{h}(t) x_{h}$ exists on $\left[t_{0}, t_{1}\right)$. The semigroups $T_{h}(t)$ are said to conditionally approximate $T(t)$ on a set $U \subset X$ uniformly on an interval $I \equiv\left[t_{0}, t_{1}\right] \subset \mathbf{R}^{+}$if there are a constant $h(I, U)>0$ and 
a function $\eta(h, I, U)$ defined for $0<h \leq h(I, U)$ such that

$$
\lim _{h \rightarrow 0} \eta(h, I, U)=0
$$

and, for any $0<h \leq h(I, U)$, if $u \in U \cap X_{h}$ has the property that $T(t) u, T_{h}(t) u$ are defined and belong to $U$ for $t \in\left[0, t_{2}\right]$ where $t_{0}<t_{2} \leq t_{1}$, then

$$
\left\|T(t) u-T_{h}(t) u\right\|_{X} \leq \eta(h, I, U) \quad \text { for } t_{0} \leq t \leq t_{2} .
$$

The semigroups $T_{h}(t)$ are said to approximate $T(t)$ on a set $U \subset X$ uniformly on an interval $I \subset \mathrm{R}^{+}$if $T_{h}(t)$ conditionally approximates $T(t)$ on $U$ uniformly on $I$ and if, moreover, for $0<h \leq h(I, U)$ and any $u \in U \cap X_{h}$, the functions $T(t) u, T_{h}(t) u$ are defined and satisfy the inequality (2.3) for all $t \in I$.

The semigroups $T_{h}(t)$ are said to (conditionally) approximate $T(t)$ on $U \subset X$ uniformly on compact sets of $\mathbf{R}^{+}$if $T_{h}(t)$ (conditionally) approximates $T(t)$ on $U$ uniformly on any compact interval $I \subset \mathbf{R}^{+}$. We recall that, in the following, $\mathcal{N}(B, \varepsilon)$ denotes the $\varepsilon$-neighborhood of a set $B$ in the Banach space $X$.

LEMMA 2.1. Assume that there exist a bounded set $B_{0} \subset X$ and an open set $U_{0} \supset \mathcal{N}\left(B_{0}, d_{0}\right)$ for some $d_{0}>0$ such that $B_{0}$ attracts $U_{0}$ under $T(t)$. Moreover, assume that there exist an open set $U_{1} \supset \mathcal{N}\left(B_{0}, d_{1}\right)$ for some $d_{1}>0$ and a constant $t_{0} \geq 0$ such that $T_{h}(t)$ approximates $T(t)$ on $U_{1}$ uniformly on compact sets of $\left[t_{0}, \infty\right)$. Then, for any $\varepsilon_{0}>0$, there are $h_{0}>0$ and $\tau_{0}>t_{0}$ such that, for $0<h \leq h_{0}$, for $t \geq \tau_{0}$,

$$
T_{h}(t)\left(U_{0} \cap U_{1} \cap X_{h}\right) \subset \mathcal{N}\left(B_{0}, \varepsilon_{0}\right) .
$$

Proof. Without any restriction, we can assume that $\varepsilon_{0} \leq \inf \left(d_{0}, d_{1}\right)$. As $B_{0}$ attracts $U_{0}$, there exists $\tau_{0}>t_{0}$ such that, for $t \geq \tau_{0}, T(t) U_{0} \subset \mathcal{N}\left(B_{0}, \varepsilon_{0} / 2\right)$. Thanks to the hypothesis (2.2), there exists $h_{0}>0$ such that, for $h \leq h_{0}, \eta\left(h,\left[t_{0}, 2 \tau_{0}\right], U_{1}\right) \leq$ $\varepsilon_{0} / 2$. Therefore, for $h \leq h_{0}$, for $\tau_{0} \leq t \leq 2 \tau_{0}, T_{h}(t)\left(U_{0} \cap U_{1} \cap X_{h}\right) \subset \mathcal{N}\left(B_{0}, \varepsilon_{0}\right)$. Let us remark that $U_{0} \cap U_{1} \cap X_{h} \neq \varnothing$, because $U_{0} \cap U_{1} \supset \mathcal{N}\left(B_{0}, \inf \left(d_{0}, d_{1}\right)\right)$.

Now, let us prove by induction that, for $t \geq \tau_{0}, T_{h}(t)\left(U_{0} \cap U_{1} \cap X_{h}\right) \subset \mathcal{N}\left(B_{0}, \varepsilon_{0}\right)$. Assume that, for $\tau_{0} \leq t \leq n \tau_{0}, T_{h}(t)\left(U_{0} \cap U_{1} \cap X_{h}\right) \subset \mathcal{N}\left(B_{0}, \varepsilon_{0}\right)$ and let us prove this property for $\tau_{0} \leq t \leq(n+1) \tau_{0}$. If $n \tau_{0} \leq t \leq(n+1) \tau_{0}$, then $t=(n-1) \tau_{0}+\tau$ with $\tau_{0} \leq \tau \leq 2 \tau_{0}$. Let $u_{0 h} \in U_{0} \cap U_{1} \cap X_{h}$; we have

$$
T_{h}(t) u_{0 h}=T_{h}(\tau) T_{h}\left((n-1) \tau_{0}\right) u_{0 h} .
$$

By the induction hypothesis, $T_{h}\left((n-1) \tau_{0}\right) u_{0 h} \in \mathcal{N}\left(B_{0}, \varepsilon_{0}\right) \cap X_{h}$, and hence, $T_{h}\left((n-1) \tau_{0}\right) u_{0 h} \in U_{0} \cap U_{1} \cap X_{h}$. Therefore, on the one hand,

$$
T(\tau) T_{h}\left((n-1) \tau_{0}\right) u_{0 h} \in \mathcal{N}\left(B_{0}, \varepsilon_{0} / 2\right),
$$

and, on the other hand,

$$
\left\|T(\tau) T_{h}\left((n-1) \tau_{0}\right) u_{0 h}-T_{h}(\tau) T_{h}\left((n-1) \tau_{0}\right) u_{0 h}\right\|_{X} \leq \varepsilon_{0} / 2 .
$$

Finally, $T_{h}(\tau) T_{h}\left((n-1) \tau_{0}\right) u_{0 h} \in \mathcal{N}\left(B_{0}, \varepsilon_{0}\right)$, for $\tau_{0} \leq \tau \leq 2 \tau_{0}$, i.e., $T_{h}(t) u_{0 h} \in$ $\mathcal{N}\left(B_{0}, \varepsilon_{0}\right)$ for $\tau_{0} \leq t \leq(n+1) \tau_{0}$.

If the dynamical system $T(t)$ has a local compact attractor $A$, the hypotheses of Lemma 2.1 can be weakened, as we shall see below. 
Proposition 2.2. Assume that there exist a compact set $A \subset X$ and an open neighborhood $N_{1}$ of $A$ such that $A$ attracts $N_{1}$. Suppose that there are constants $h_{0}>0, \delta_{0}>0, t_{0} \geq 0$ and two open neighborhoods $N_{2}, N_{3}$ of $A$, with $N_{1} \subset N_{2} \subset$ $\mathcal{N}\left(N_{2}, \delta_{0}\right) \subset N_{3}$, such that, for $0<h \leq h_{0}$,

(i) $T(t) N_{1} \subset N_{2}$ for $t \geq 0$,

(ii) $T_{h}(t)\left(N_{1} \cap X_{h}\right) \subset N_{2}$ for $0 \leq t \leq t_{0}$,

(iii) for any $x_{h} \in \mathcal{N}\left(N_{2}, \delta_{0}\right) \cap X_{h}$, there exists $t\left(x_{h}\right)>0$ such that $T_{h}(t) x_{h} \in N_{3}$ for $0 \leq t \leq t\left(x_{h}\right)$.

Also assume that $T_{h}(t)$ conditionally approximates $T(t)$ on $N_{3}$ uniformly on compact sets of $\left[t_{0}, \infty\right)$. Then, for any $\varepsilon_{0}>0$, there are $\bar{h}>0$ and $\tau_{0}>t_{0}$ such that, for $0<h \leq \bar{h}$ and $t \geq \tau_{0}$,

$$
T_{h}(t)\left(N_{1} \cap X_{h}\right) \subset \mathcal{N}\left(A, \varepsilon_{0}\right) .
$$

Proof. As $T_{h}(t)$ conditionally approximates $T(t)$ on $N_{3}$ uniformly on compact sets of $\left[t_{0},+\infty\right)$, for any $t_{1}>t_{0}$, there is a positive number $\bar{h}\left(t_{1}\right)$ so that $\eta\left(h,\left[t_{0}, t_{1}\right], N_{3}\right)<\delta_{0} / 4$ for $h \leq \bar{h}\left(t_{1}\right)$. For any $x_{h} \in N_{1} \cap X_{h}$ and any $t, t_{0} \leq t \leq t_{1}$, we want to prove that $T_{h}(t) x_{h} \in N_{3}$, because this will show that

$$
\left\|T(t) x_{h}-T_{h}(t) x_{h}\right\|_{X} \leq \eta\left(h,\left[t_{0}, t_{1}\right], N_{3}\right)
$$

for $t_{0} \leq t \leq t_{1}$ and we may apply Lemma 2.1. Assume this is not the case. Then, by (ii) and (iii), there exists $t_{2}, t_{0}<t_{2} \leq t_{1}$, such that $T_{h}(t) x_{h} \in N_{3}$ for $0 \leq t<t_{2}$ and $T_{h}\left(t_{2}\right) x_{h} \notin N_{3}$. But then $T_{h}(t) x_{h} \in \mathcal{N}\left(N_{2}, \delta_{0} / 4\right)$ for $0 \leq t<t_{2}$ and hence $T_{h}\left(t_{2}\right) x_{h} \in \mathcal{N}\left(N_{2}, \delta_{0} / 2\right)$, which is a contradiction. This proves the proposition.

Remark 2.3. If $A$ is a local, compact attractor under the semigroup $T(t)$, then $A$ is stable, and there always exist neighborhoods $N_{1}, N_{2}$ satisfying (i) in Proposition 2.2 .

To state the next result, we need some additional terminology. Following Hale, LaSalle and Slemrod [13] or Hale and Lopes [14], a semigroup $T(t), t \geq 0$, on a Banach space $X$ is said to be asymptotically smooth if, for any bounded set $B \subset X$, there is a compact set $J=J(B) \subset X$ such that $J$ attracts the set $\{x \in B: T(t) x \in B$ for $t \geq 0\}$. A special case of asymptotically smooth semigroups are $\alpha$-contracting semigroups (see Hale and Lopes [14]); $T(t)$ is an $\alpha$-contracting semigroup if $T(t)=S(t)+U(t)$, where $U(t), t>0$, is completely continuous and $S(t), t \geq 0$, is a bounded linear operator for which there is a $\beta>0$ such that $\|S(t)\|_{\mathcal{L}(X ; X)} \leq \exp (-\beta t), t \geq 0$.

The next result gives conditions for the existence of compact attractors $A_{h}$ for $T_{h}(t)$ and the upper semicontinuity of these sets "at $h=0$ ".

THEOREM 2.4. Assume that $T(t)$ has a local, compact attractor $A$ and that the hypotheses of Proposition 2.2 are satisfied. If each $T_{h}(t)$ is asymptotically smooth, then there is $h_{0}>0$ such that, for $0<h \leq h_{0}, T_{h}(t)$ admits a local, compact attractor $A_{h}$, which attracts $N_{1} \cap X_{h}$. Moreover, $\delta_{X}\left(A_{h}, A\right) \rightarrow 0$ as $h \rightarrow 0$.

Proof. From Proposition 2.2, it follows that $T_{h}(t)\left(N_{1} \cap X_{h}\right), t \geq 0$, belongs to a bounded set in $X_{h}$. The results in Hale, LaSalle and Slemrod [13] (see also [12]) imply the existence of a compact attractor $A_{h}$ for $T_{h}(t)$ which attracts $N_{1} \cap X_{h}$. Owing to Relation (2.4), we can take $A_{h} \subset N\left(A, \varepsilon_{0}\right)$. Since $\varepsilon_{0}$ is arbitrary, we obtain the result. 
COROLLARY 2.5. Assume that $T(t)$ has a local compact attractor $A$ and that the conditions of Proposition 2.2 are satisfied. If each space $X_{h}$ is finite-dimensional, the conclusions of Theorem 2.4 hold.

In the general case, the hypotheses of Theorem 2.4 do not enable us to give any information about the distance $\delta_{X}\left(A, A_{h}\right)$. However, if $A$ is reduced to a point $x_{0}$, then, of course, under the hypotheses of Theorem $2.4, \delta\left(A, A_{h}\right) \rightarrow 0$.

In the next result, we assume the attractors for the approximate semigroups exist and conclude that the original semigroup admits an attractor.

PROPOSITION 2.6. Suppose there are bounded open sets $N_{1} \subset N_{2} \subset X$ and positive constants $\varepsilon_{0}, \bar{\varepsilon}_{0}, h_{0}, t_{0}, \delta_{0}$ such that, for each $0<h \leq h_{0}$, the semigroup $T_{h}(t)$ has a local compact attractor $A_{h} \subset X$, with $\mathcal{N}\left(A_{h}, \bar{\varepsilon}_{0}\right) \subset N_{1}$, and that

(i) $A_{h}$ attracts $N_{1}$ uniformly, that is, for any $\varepsilon_{1}>0$, there is a $\tau_{1}>0$, independent of $h$, such that $T_{h}(t)\left(N_{1} \cap X_{h}\right) \subset \mathcal{N}\left(A_{h}, \varepsilon_{1}\right)$ for $t \geq \tau_{1}$,

(ii) $T_{h}(t)\left(N_{1} \cap X_{h}\right) \subset N_{2} \cap X_{h}$, for all $t \geq 0$,

(iii) $T(t) N_{1} \subset N_{2}$ for $0 \leq t \leq t_{0}$,

(iv) $T(t) x$ is well defined for $x \in \mathcal{N}\left(N_{2}, \varepsilon_{0}\right)$ for $0 \leq t \leq \delta_{0}$.

Also assume that $T_{h}(t)$ conditionally approximates $T(t)$ on $\mathcal{N}\left(N_{2}, \varepsilon_{0}\right)$ uniformly on compact sets of $\left[t_{0},+\infty\right)$. Then, there exists $\tau \geq t_{0}$ such that, for $t \geq \tau$,

$$
T(t) N_{1} \subset N_{1} \text {. }
$$

If, in addition, $T(t)$ is asymptotically smooth, then $T(t)$ has a local compact attractor $A$ attracting $N_{1}$ and, for any $\varepsilon>0$, there exists $h_{1}>0$ such that, for $0<h \leq h_{1}$,

$$
A \subset \mathcal{N}\left(A_{h}, \varepsilon\right) .
$$

Proof. Let us first show that

$$
T(t) N_{1} \subset \mathcal{N}\left(N_{2}, \varepsilon_{0}\right) \quad \text { for all } t \geq 0 .
$$

Owing to (iii), $T(t) N_{1} \subset \mathcal{N}\left(N_{2}, \varepsilon_{0}\right)$ for $0 \leq t \leq t_{0}$. Suppose that the property (2.7) is not true; then there exist $x \in N_{1}$ and $t_{2}>t_{0}$ such that $T\left(t_{2}\right) x \in \partial\left(\mathcal{N}\left(N_{2}, \varepsilon_{0}\right)\right)$ and $T(t) x \in \mathcal{N}\left(N_{2}, \varepsilon_{0}\right)$ for $0 \leq t<t_{2}$ (the existence of $t_{2}$ is ensured by (iv)). Thanks to the approximation property (2.1), there exist a positive number $h_{2}$ and, for $0<h \leq h_{2}$, an element $x_{h} \in N_{1} \cap X_{h}$ close enough to $x$ so that, for $0 \leq t \leq t_{2}, 0<h \leq h_{2}$,

$$
\left\|T(t) x_{h}-T(t) x\right\|_{X}<\varepsilon_{0} / 3 .
$$

Moreover, there exists $h_{3}>0$, with $h_{3} \leq \inf \left(h_{0}, h_{2}\right)$, such that, for $0<h \leq h_{3}$,

$$
\eta\left(h,\left[t_{0}, t_{2}\right], \mathcal{N}\left(N_{2}, \varepsilon_{0}\right)\right)<\varepsilon_{0} / 3 .
$$

Thus, since $T_{h}(t)$ conditionally approximates $T(t)$ on $\mathcal{N}\left(N_{2}, \varepsilon_{0}\right)$ uniformly on $\left[t_{0}, t_{2}\right]$, (2.8), (2.9) and (ii) imply that $T\left(t_{2}\right) x \in \mathrm{Cl}\left(\mathcal{N}\left(N_{2}, 2 \varepsilon_{0} / 3\right)\right)$, which is a contradiction. Thus (2.7) is true.

Next we show that $T(t) N_{1} \subset N_{1}$ for $t \geq \tau$, where $\tau \geq t_{0}$ is a constant. Owing to the property (i), there exists $\tau \geq t_{0}$ such that, for $0<h \leq h_{0}, T_{h}(t)\left(N_{1} \cap X_{h}\right) \subset$ $\mathcal{N}\left(A_{h}, \bar{\varepsilon}_{0} / 4\right)$ for $t \geq \tau$. Now let $x \in N_{1}$ be given. As above, there exist a positive 
number $h_{4}$, with $h_{4} \leq h_{0}$, and, for $0<h \leq h_{4}$, an element $x_{h} \in N_{1} \cap X_{h}$ close to $x$ such that, for $0<h \leq h_{4}$,

$$
\left\|T(t) x_{h}-T(t) x\right\|_{X}<\bar{\varepsilon}_{0} / 3 \text { for all } t, \text { with } 0 \leq t \leq 2 \tau
$$

and

$$
\eta\left(h,\left[t_{0}, 2 \tau\right], \mathcal{N}\left(N_{2}, \varepsilon_{0}\right)\right)<\bar{\varepsilon}_{0} / 3 .
$$

As (2.7) holds and $T_{h}(t)$ conditionally approximates $T(t)$ on $\mathcal{N}\left(N_{2}, \varepsilon_{0}\right)$ uniformly on $\left[t_{0}, 2 \tau\right]$, we derive from (2.10) and (2.11) that $T(t) x \in \mathcal{N}\left(A_{h}, 3 \bar{\varepsilon}_{0} / 4\right) \subset N_{1}$, for $\tau \leq t \leq 2 \tau$.

An easy induction, similar to the one of the proof of Lemma 2.1, shows that $T(t) x \in N_{1}$, for $t \geq \tau$.

If, moreover, $T(t)$ is asymptotically smooth, we conclude, by using a result of [7], that $T(t)$ has a compact attractor $A \subset N_{1}$ attracting $N_{1}$. It remains to prove (2.6). Let $\varepsilon>0$ be given. By (i), there exists $\tau_{1}>t_{0}$, independent of $h$, such that $T_{h}(t)\left(N_{1} \cap X_{h}\right) \subset \mathcal{N}\left(A_{h}, \varepsilon / 3\right)$ for $t \geq \tau_{1}$ and for $0<h \leq h_{0}$. Because of the compactness of $A$, there exists $h_{5}, 0<h_{5} \leq h_{0}$, such that, for $0<h \leq h_{5}$, with each element $x \in A$ we can associate an element $P_{h} x$ in $N_{1} \cap X_{h}$ such that

$$
\left\|T(t) x-T(t) P_{h} x\right\|_{X} \leq \varepsilon / 3 \quad \text { for } 0 \leq t \leq \tau_{1} .
$$

Finally, there exists $h_{1}, 0<h_{1} \leq h_{5}$, such that, for any $x_{h} \in N_{1} \cap X_{h}$,

$$
\left\|T(t) x_{h}-T_{h}(t) x_{h}\right\|_{X} \leq \varepsilon / 3 \quad \text { for } t_{0} \leq t \leq \tau_{1} .
$$

Thus, for $0<h \leq h_{1}, T\left(\tau_{1}\right) x \in \mathcal{N}\left(A_{h}, \varepsilon\right)$ for all $x$ in $A$; and from the equality $T\left(\tau_{1}\right) \mathcal{A}=A$ we deduce the inclusion (2.6).

Remark 2.7. Property (2.6) means that $\delta_{X}\left(A, A_{h}\right) \rightarrow 0$ as $h \rightarrow 0$. Let us remark that, under the hypotheses of Proposition 2.6, $\delta_{X}\left(A_{h}, A\right)$ also tends to 0 as $h$ tends to 0 . Indeed, as $A$ attracts $N_{1}$, for any $\varepsilon_{1}>0$, there exists $\tau_{1}>t_{0}$ such that $T(t) A_{h} \subset \mathcal{N}\left(A, \varepsilon_{1} / 2\right)$ for $t \geq \tau_{1}$. On the other hand, there exists $\bar{h}>0$ such that, for $0<h \leq \bar{h}, \eta\left(h,\left[t_{0}, \tau_{1}\right], \mathcal{N}\left(N_{2}, \varepsilon_{0}\right)\right) \leq \varepsilon_{1} / 2$. Thus, $T_{h}\left(\tau_{1}\right) A_{h} \subset \mathcal{N}\left(A, \varepsilon_{1}\right)$ and, since $T_{h}\left(\tau_{1}\right) A_{h}=A_{h}, A_{h} \subset \mathcal{N}\left(A, \varepsilon_{1}\right)$ for $0<h \leq \bar{h}$.

Remark 2.8. The assumption (i) in Proposition 2.6 that $A_{h}$ attracts $N_{1}$ uniformly is a very strong condition. However, one would expect numerical procedures to have such a property. The detailed structure of the flow on the attractor $A_{h}$ could vary considerably with $h$. This depends on the flow defined by $T(t)$. Consider, for example, a scalar equation $\dot{u}=f(u), u \in R$, where the flow is given by $\longrightarrow \longrightarrow \longrightarrow$. If one approximates this flow numerically, two situations could arise. One could obtain either the approximate flow $\rightarrow \bullet \leftarrow$ or $\longrightarrow \leftarrow$ The global attractor in one case is a point and in the other is a line segment. The global attractor for the original problem is a line segment. For one of the approximation schemes, the attractors $A_{h}$ approach a point as $h \rightarrow 0$ which is a local attractor for $T(t)$ and, for the other, $A_{h}$ approaches a line segment which is the global attractor for $T(t)$. If the flow on the attractor for $T(t)$ is less sensitive to small perturbations, this situation will not arise.

Let us now turn to the question of how close $A_{h}$ is to $A$ with the measure of closeness given by $\delta_{X}\left(A_{h}, A\right)$. We give some results in this direction for some particular cases. 
Proposition 2.9. Suppose the hypotheses of Theorem 2.4 are satisfied with the associated function $\eta\left(h, I, N_{3}\right)=c h^{\gamma_{0}}$ for some positive constants $c, \gamma_{0}$, independent of $h$ and $I \subset\left[t_{0}, \infty\right)$. Then there is a constant $c_{1}>0$ such that $\delta_{X}\left(A_{h}, A\right) \leq c_{1} h^{\gamma_{0}}$ for $0<h \leq h_{0}$.

Proof. The proof follows from the proof of Proposition 2.2 and Theorem 2.4 using the special function $\eta\left(h, I, N_{3}\right)=c h^{\gamma_{0}}$.

The hypothesis on $\eta\left(h, I, N_{3}\right)$ in Proposition 2.9 is not usually satisfied. A more reasonable condition on $\eta\left(h, I, N_{3}\right)$ is given in the next result, but then we must impose stronger attractivity properties of $A$.

PROPOSITION 2.10. Assume the hypotheses of Theorem 2.4 are satisfied with the associated function $\eta\left(h,\left[t_{0}, t_{1}\right], N_{3}\right)=c_{0} h^{\gamma_{0}} e^{\alpha_{0} t_{1}}$ for some positive constants $c_{0}, \gamma_{0}, \alpha_{0}$, independent of $h$ and $t_{1}$. If there are an open neighborhood $U$ of $A$ and positive constants $c_{1}, \beta_{0}$ such that

$$
\delta_{X}(T(t) U, A) \leq c_{1} e^{-\beta_{0} t}, \quad t \geq 0,
$$

then, for $h \leq h_{0}$, we have

$$
\delta_{X}\left(A_{h}, A\right) \leq \operatorname{ch}^{\gamma_{0} \beta_{0} /\left(\alpha_{0}+\beta_{0}\right)}
$$

for some positive constant $c$.

Proof. If

$$
t_{1}=-\frac{1}{\beta_{0}} \log \frac{c_{0}}{c_{1}} h^{\gamma_{0} \beta_{0} /\left(\alpha_{0}+\beta_{0}\right)},
$$

then $\delta_{X}(T(t) U, A) \leq c_{0} h^{\gamma_{0} \beta_{0} /\left(\alpha_{0}+\beta_{0}\right)}$ for $t \geq t_{1}$. Since $A_{h}$ is invariant, for any $x_{h} \in A_{h}$, there is a $y_{h} \in A_{h}$ such that $x_{h}=T_{h}\left(t_{1}\right) y_{h}$. If $x=T\left(t_{1}\right) y_{h}$, then

$$
\left\|x_{h}-x\right\|_{X}=\left\|T_{h}\left(t_{1}\right) y_{h}-T\left(t_{1}\right) y_{h}\right\|_{X} \leq c_{0}^{1-\alpha_{0} / \beta_{0}} c_{1} h^{\gamma_{0} \beta_{0} /\left(\alpha_{0}+\beta_{0}\right)} .
$$

This completes the proof.

Remark 2.11. If $T(t)$ is a gradient system (for the definition, see Hale [12]) for which there is a $t_{1}>0$ such that $T(t)$ is either compact for $t>t_{1}$ or an $\alpha$-contraction, and if the set of equilibrium points $E$ (i.e., the points $\phi$ such that $T(t) \phi=\phi, t \geq 0)$ is bounded, then we know that $T(t)$ has a compact attractor $A$. If, in addition, each element of $E$ is hyperbolic, then $E$ is a finite set, $\operatorname{dim} W^{u}(\phi)<+\infty$ and $A=\bigcup_{\phi \in E} W^{u}(\phi)$, where $W^{u}(\phi)$ is the unstable set of $\phi$. Furthermore, if the stable and unstable manifolds intersect transversally, there is an open neighborhood $U$ of $A$ such that $\delta_{X}(T(t) U, A) \rightarrow 0$ exponentially as $t \rightarrow+\infty$.

Thus, if the approximate semigroups $T_{h}(t)$ satisfy the hypothesis of Theorem 2.4 with $\eta\left(h,\left[t_{0}, t_{1}\right], N_{3}\right)=c_{0} h^{\gamma_{0}} e^{\alpha_{0} t_{1}}, T_{h}(t)$ admits a local compact attractor $A_{h}$ for $h$ small enough and, by Proposition 2.10, we obtain a good estimate of $\delta_{X}\left(A_{h}, A\right)$.

Now assume that, for $h>0, T_{h}(t)$ is a gradient system. Then, one can prove that, for $h$ small enough, the set of equilibrium points $E_{h}$ of $T_{h}$ is finite and has the same cardinality as $E$, and one can give an estimate of $\delta_{X}\left(E, E_{h}\right)$ and $\delta_{X}\left(E_{h}, E\right)$. Moreover, $A_{h}=\bigcup_{\phi_{h} \in E_{h}} W^{u}\left(\phi_{h}\right)$, where $W^{u}\left(\phi_{h}\right)$ is the unstable set of $\phi_{h}$. (For more details, see Lin and Raugel [25]).

In Remark 2.11 we have encountered a situation where the conditions of Proposition 2.10 are satisfied. One would expect that the hypothesis in Proposition 2.10 
that $T(t) U \rightarrow A$ exponentially as $t \rightarrow+\infty$ will be satisfied in specific evolutionary problems, at least generically with respect to the vector fields. A more precise statement is needed and certainly is nontrivial.

Let us end this section by pointing out that in some cases the semigroups $T_{h}(t)$ do not conditionally approximate $T(t)$ on any open set $U \subset X$. In this case, one has to use other ways to prove that $T_{h}(t)$ admits a local compact attractor $A_{h}$ for $h$ small enough. In Subsection 6.2 we shall encounter a typical example of this case.

\section{Galerkin Approximation of Sectorial Evolutionary Equations.}

3.1. Galerkin Approximation of a Parabolic Equation. Let $V$ and $H$ be two (real) Hilbert spaces such that $V$ is included in $H$ with a continuous and dense imbedding; the space $H$ is identified with its dual space, and the inner product of $H$, as well as the duality pairing between $V$ and its dual space $V^{\prime}$, is denoted by $(\cdot, \cdot)$ (so we have the inclusions $V \subset H \subset V^{\prime}$, where the imbeddings are continuous and dense). We introduce a continuous, bilinear form on $V \times V:(u, v) \in V \times V \rightarrow a(u, v)$ and the corresponding operator $A \in \mathcal{L}\left(V ; V^{\prime}\right)$ defined by

$$
\forall u, v \in V, \quad a(u, v)=(A u, v) .
$$

We denote by $C_{0}$ the constant of continuity of the bilinear form $a(\cdot, \cdot)$. We also suppose that there are two constants $\gamma>0$ and $\gamma_{0} \geq 0$ such that

$$
\forall v \in V, \quad a(v, v)+\gamma_{0}\|v\|_{H}^{2} \geq \gamma\|v\|_{V}^{2} .
$$

Moreover, if

$$
b(u, v)=a(u, v)-a(v, u),
$$

we assume that there exists a constant $C_{1}>0$ such that

$$
|b(u, v)| \leq C_{1}\|u\|_{V}\|v\|_{H}
$$

Now we consider the nonliner equation

$$
\left\{\begin{array}{l}
d u / d t+A u=f(u), \\
u(0)=u_{0}
\end{array}\right.
$$

where $u_{0}$ belongs to $V$ and $f: V \rightarrow H$ is locally Lipschitz continuous (i.e., $f$ is continuous and, for any bounded set $B$ of $V$, there is a constant $k_{B}$ such that $\|f(u)-f(v)\|_{H} \leq k_{B}\|u-v\|_{V}$ for $u, v$ in $\left.B\right)$.

Remark 3.1. We may always assume that $\gamma_{0}=0$. If $\gamma_{0}>0$, we can set $A_{1}=$ $A+\gamma_{0} I$ and replace Eq. (3.3) by

$$
\left\{\begin{array}{l}
d u / d t+A_{1} u=f(u)+\gamma_{0} u, \\
u(0)=u_{0} .
\end{array}\right.
$$

Therefore, we assume in the sequel that $\gamma_{0}=0$.

Let $D(A)=\{v \in V ; A v \in H\}$. Note that $D(A)$ is dense in $V$ and $H$ and that $A$ is a sectorial operator on $H$ in the sense of Henry [17], so that we can define the operators $A^{\alpha}$ for any $\alpha \geq 0$. (We recall that $A$ is sectorial if and only if the semigroup $e^{-A t}$ generated by $A$ is an analytic semigroup.) If we define $X^{\alpha}=D\left(A^{\alpha}\right), \alpha \geq 0$, with the graph norm $\|v\|_{X^{\alpha}}=\left\|A^{\alpha} v\right\|_{H}, v \in X^{\alpha}$, then $X^{\alpha}$ is a Hilbert space normed by $\|v\|_{X^{\alpha}}$ and $X^{0}=H$ (for more details, see Henry [17, pp. 26-29]). From the hypothesis (3.2) we derive at once that $D(A)=D\left(A^{*}\right)$, where 
$A^{*}$ is the adjoint operator of $A$, defined by $\left(A^{*} u, v\right)=a(v, u)$ for any $u, v$ in $V$. By using a result of Lions ([26, Théorème 5.3]) we conclude that

$$
D\left(A^{1 / 2}\right)=D\left(A^{* 1 / 2}\right)=V .
$$

Thus we are in the context considered in [17, Chapter 3], so that we can use existence, uniqueness and regularity results for the solution of the equation (3.3). In particular, we know that under the above hypotheses on $A, f$ and $u_{0}$ there is a unique solution in $V$ of Eq. (3.3) on a maximal interval of existence $\left(0, \tau_{u_{0}}\right)$. Here we assume that all solutions are defined for $t \geq 0$, so that we can introduce the map $T(t): V \rightarrow V, t \geq 0$, defined by $T(t) u_{0}=u\left(t, u_{0}\right)$ and obtain a $C^{0}$-semigroup on $V$. We also suppose that $T(t)$ has a (local) compact attractor $A$ which attracts a bounded open set $O, O \supset A$ (see [12] for the existence of $A$ ).

Now let us turn to a finite-dimensional approximation of Eq. (3.3). Let $h>0$ be a real parameter which will tend to 0 and $\left(V_{h}\right)_{h}$ a family of finite-dimensional subspaces of $V$. We introduce the operator $A_{h} \in \mathcal{L}\left(V_{h} ; V_{h}\right)$ defined by

$$
\forall v_{h} \in V_{h}, \quad\left(A_{h} w_{h}, v_{h}\right)=a\left(w_{h}, v_{h}\right) \quad \text { for } w_{h} \text { in } V_{h} .
$$

Let $Q_{h} \in \mathcal{L}\left(H ; V_{h}\right)$ be the projector on $V_{h}$ in the space $H$, i.e.,

$$
\forall v \in H, \forall v_{h} \in V_{h}, \quad\left(v-Q_{h} v, v_{h}\right)=0,
$$

and let $P_{h} \in \mathcal{L}\left(V ; V_{h}\right)$ be the projector on $V_{h}$ in the space $V$, i.e.,

$$
\forall v \in V, \forall v_{h} \in V_{h}, \quad a\left(v-P_{h} v, v_{h}\right)=0 .
$$

Now consider the following equation in $V_{h}$ :

$$
\left\{\begin{array}{l}
d u_{h} / d t+A_{h} u_{h}=Q_{h} f\left(u_{h}\right), \\
u_{h}(0)=u_{0 h},
\end{array}\right.
$$

where $u_{0 h} \in V_{h}$. Equation (3.3) $)_{h}$ is an ordinary differential equation. We introduce the map $T_{h}(t): V_{h} \rightarrow V_{h}$, defined by $T_{h}(t) u_{0 h}=u_{h}\left(t, u_{0 h}\right)$ as long as $u_{h}\left(t, u_{0 h}\right)$ exists. $T_{h}(t) u_{0 h}$ is continuous in $t$ and $u_{0 h}$ when it is well defined and, if $T_{h}(t) u_{0 h}$ exists on $\left[t_{0}, t_{1}\right)$, it is left-continuous at $t_{1}$.

In order to prove that $T_{h}(t)$ also admits a compact attractor $A_{h}$, for $h$ small enough, we need the following additional hypotheses on the spaces $\left(V_{h}\right)_{h}$ :

There exist an integer $m>0$ and, for any $\beta, \frac{1}{2} \leq \beta \leq 1$, a constant $C(\beta)>0$ such that, for all $w$ in $X^{\beta} \equiv D\left(A^{\beta}\right)$,

$$
\left\|w-P_{h} w\right\|_{V}+\left\|w-Q_{h} w\right\|_{V} \leq C(\beta) h^{2 m(\beta-1 / 2)}\|w\|_{X^{\beta}}
$$

and

$$
\left\|w-P_{h} w\right\|_{H}+\left\|w-Q_{h} w\right\|_{H} \leq C(\beta) h^{2 m \beta}\|w\|_{X^{\beta}} .
$$

Remark 3.2. The hypotheses (3.6) (i) and (3.6) (ii) are realistic and are satisfied in many cases when $A$ is an elliptic differential operator (see Ciarlet [5] and Example 3.1 below). We point out that in this case the quantity $2 m$ in (3.6) is rather related to the order of the diffrential operator $A$ than to the order of the "approximation" of $V$ by $V_{h}$. In Example 3.1 below, $2 m$ remains equal to 2 (which is the order of $\mathcal{L})$, even if we replace $P_{1}(K)$ in (3.8) by a space of higher-order polynomials. 
Example 3.1. Let $\Omega$ be a regular bounded domain or a convex bounded set in $\mathbf{R}^{2}$. In $\Omega$ we are given an elliptic operator of the following form:

$$
\mathcal{L} v=\sum_{i, j=1}^{2} a_{i j}(x) \frac{\partial^{2} v}{\partial x_{i} \partial x_{j}}+\sum_{j=1}^{2} b_{j}(x) \frac{\partial v}{\partial x_{j}}+c(x) v
$$

where the coefficients $a_{i j}, b_{j}, c$ are smooth enough and where $\mathcal{L}$ is assumed to be uniformly and strongly elliptic. If $A$ denotes the operator $-\mathcal{L}$, with homogeneous Dirichlet boundary conditions, then the hypotheses (3.1) and (3.4) are satisfied with $D(A)=H^{2}(\Omega) \cap H_{0}^{1}(\Omega), D\left(A^{1 / 2}\right)=V=H_{0}^{1}(\Omega), H=L^{2}(\Omega)$. And one can find finite-dimensional subspaces $V_{h}$ of $H_{0}^{1}(\Omega)$ such that the conditions (3.6) (i), (3.6) (ii) are satisfied with $m=1$. For instance, if $\Omega$ is a convex polygonal domain, we introduce a uniformly regular family $\left(\tau_{h}\right)_{h}$ of triangulations in the sense of [5], where $\tau_{h}$ is made of triangles with diameters bounded by $h$. And we set

$$
V_{h}=\left\{v_{h} \in C^{0}(\bar{\Omega}) \cap H_{0}^{1}(\Omega): \forall K \in \tau_{h},\left.v_{h}\right|_{K} \in P_{1}(K)\right\},
$$

where $P_{1}(K)$ is the space of all polynomials of degree $\leq 1$ on $K$. In this case, the hypotheses (3.6) (i) and (3.6) (ii) are satisfied with $m=1$. Moreover, even if the family $\left(\tau_{h}\right)_{h}$ is only regular, the hypothesis (3.6) (ii) is satisfied and the condition (3.6) (i) usually holds (see Crouzeix and Thomée [9]).

THEOREM 3.1. Under the above hypotheses, there exists $h_{0}>0$ such that, for $h \leq h_{0}, T_{h}(t)$ admits a local compact attractor $A_{h}$, which attracts an open set $N_{1} \cap V_{h}$, where $N_{1}$ is independent of $h$. Moreover, $\delta_{V}\left(A_{h}, A\right) \rightarrow 0$ as $h \rightarrow 0$.

Proof. We shall prove that the hypotheses of Proposition 2.2 are satisfied by $T(t)$ and $T_{h}(t)$ for $h$ small enough. Clearly, it is sufficient to show that there are constants $h_{0}>0, \delta_{0}>0$ and $t_{0}>0$ and three open neighborhoods $N_{1}, N_{2}, N_{3}$ of $A$ with $N_{1} \subset \mathcal{O}, N_{1} \subset N_{2} \subset \mathcal{N}\left(N_{2}, \delta_{0}\right) \subset N_{3}$, such that the conditions (i) and (ii) of Proposition 2.2 are satisfied and that $T_{h}(t)$ conditionally approximates $T(t)$ on $N_{3}$ uniformly on compact sets of $\left[t_{0},+\infty\right)$. Let us prove this in two steps.

First step. As $A$ is a compact attractor, there is a bounded open neighborhood $N_{1}$ of $A$ such that $N_{1} \subset 0$ and $T(t) N_{1} \subset N_{1}$ for $t \geq 0$. We choose a real number $\varepsilon_{0} \geq 8 B_{0} C_{0} / \gamma$, where $B_{0}=\max _{v \in N_{1}}\|v\|_{V}$, and we set $N_{2}=\mathcal{N}\left(N_{1}, \varepsilon_{0}\right)$. Finally, let $\delta_{0}$ be a positive real number and define $N_{3}=\mathcal{N}\left(N_{2}, \delta_{0}\right)$. Now we want to prove that there exists a constant $t_{0}>0$ such that $T_{h}(t)\left(N_{1} \cap V_{h}\right) \subset N_{2}$ for $0 \leq t \leq t_{0}$. Using classical arguments of the theory of differential equations, we easily see that it is sufficient to prove the following property:

$$
\left\{\begin{array}{l}
\text { There exists a constant } t_{0}>0 \text { independent of } h \text { such that, for } \\
\text { any } u_{0 h} \in N_{1} \cap V_{h}, \text { if } T_{h}(t) u_{0 h} \text { belongs to } \mathcal{N}\left(u_{0 h}, \varepsilon_{0}+\delta_{0}\right) \text { for } \\
0 \leq t \leq t\left(u_{0 h}\right), \text { where } t\left(u_{0 h}\right) \leq t_{0}, \text { then } T_{h}(t) u_{0 h} \in \mathcal{N}\left(u_{0 h}, \varepsilon_{0}\right) \\
\text { for } 0 \leq t \leq t\left(u_{0 h}\right) .
\end{array}\right.
$$

As $f$ is globally Lipschitz continuous on $\mathcal{N}\left(N_{3}, \delta_{0}\right)$, there exist constants $M_{1}>0$ and $L>0$ such that

$$
\forall v \in \mathcal{N}\left(N_{3}, \delta_{0}\right), \quad\|f(v)\|_{H} \leq M_{1}
$$

and

$$
\forall v, w \in \mathcal{N}\left(N_{3}, \delta_{0}\right), \quad\|f(v)-f(w)\|_{H} \leq L\|v-w\|_{V}
$$


If $u_{h}$ is the solution of Eq. $(3.3)_{h}, u_{h}-u_{0 h}$ satisfies the equation

$$
d\left(u_{h}-u_{0 h}\right) / d t+A_{h}\left(u_{h}-u_{0 h}\right)=Q_{h} f\left(u_{h}\right)+A_{h} u_{0 h} .
$$

Taking the inner product in $H$ of the equation (3.10) by $d\left(u_{h}-u_{0 h}\right) / d t$, we obtain

$$
\begin{array}{r}
\left\|\frac{d}{d t}\left(u_{h}-u_{0 h}\right)\right\|_{H}^{2}+a\left(u_{h}-u_{0 h}, \frac{d}{d t}\left(u_{h}-u_{0 h}\right)\right) \\
=\left(f\left(u_{h}\right), \frac{d}{d t}\left(u_{h}-u_{0 h}\right)\right)+\frac{d}{d t} a\left(u_{0 h}, u_{h}-u_{0 h}\right) .
\end{array}
$$

But

$$
\begin{aligned}
a\left(u_{h}-u_{0 h}, \frac{d}{d t}\left(u_{h}-u_{0 h}\right)\right)= & \frac{1}{2} \frac{d}{d t} a\left(u_{h}-u_{0 h}, u_{h}-u_{0 h}\right) \\
& +\frac{1}{2} b\left(u_{h}-u_{0 h}, \frac{d}{d t}\left(u_{h}-u_{0 h}\right)\right),
\end{aligned}
$$

so that we deduce from (3.11) and (3.12), by using the inequality (3.2), that

$$
\begin{aligned}
& \left\|\frac{d}{d t}\left(u_{h}-u_{0 h}\right)\right\|_{H}^{2}+\frac{1}{2} \frac{d}{d t} a\left(u_{h}-u_{0 h}, u_{h}-u_{0 h}\right) \\
& \leq M_{1}\left\|\frac{d}{d t}\left(u_{h}-u_{0 h}\right)\right\|_{H}+C_{1}\left\|u_{h}-u_{0 h}\right\|_{V}\left\|\frac{d}{d t}\left(u_{h}-u_{0 h}\right)\right\|_{H} \\
& \quad+\frac{d}{d t} a\left(u_{0 h}, u_{h}-u_{0 h}\right),
\end{aligned}
$$

which implies that

(3.13) $\frac{d}{d t} a\left(u_{h}-u_{0 h}, u_{h}-u_{0 h}\right) \leq M_{1}^{2}+C_{1}^{2}\left\|u_{h}-u_{0 h}\right\|_{V}^{2}+2 \frac{d}{d t} a\left(u_{0 h}, u_{h}-u_{0 h}\right)$.

Finally, integrating (3.13) from 0 to $t_{h}$ and using (3.1) (with $\gamma_{0}=0$ ) and the inequality $a b \leq a^{2} / 2 \varepsilon+\varepsilon b^{2} / 2$, we obtain

$$
\left\|u_{h}\left(t_{h}\right)-u_{0 h}\right\|_{V}^{2} \leq \frac{2 t_{h} M_{1}^{2}}{\gamma}+\frac{2 C_{1}^{2}}{\gamma} \int_{0}^{t_{h}}\left\|u_{h}(s)-u_{0 h}\right\|_{V}^{2} d s+\frac{4 C_{0}^{2}}{\gamma^{2}}\left\|u_{0 h}\right\|_{V}^{2} .
$$

Thanks to Gronwall's inequality, we derive from the above estimate that

$$
\left\|u_{h}\left(t_{h}\right)-u_{0 h}\right\|_{V}^{2} \leq\left(\frac{2 t_{h} M_{1}^{2}}{\gamma}+\frac{4 C_{0}^{2}}{\gamma^{2}}\left\|u_{0 h}\right\|_{V}^{2}\right) e^{\left(2 C_{1}^{2} / \gamma\right) t_{h}}
$$

If $u_{0 h} \in N_{1} \cap V_{h},(3.14)$ becomes

$$
\left\|u_{h}\left(t_{h}\right)-u_{0 h}\right\|_{V}^{2} \leq\left(\frac{2 t_{h} M_{1}^{2}}{\gamma}+\frac{4 C_{0}^{2}}{\gamma^{2}} B_{0}^{2}\right) e^{\left(2 C_{1}^{2} / \gamma\right) t_{h}} .
$$

From (3.15) it is clear that there exists a constant $t_{0}>0$ independent of $h$ such that property (A) holds.

It remains to prove that $T_{h}(t)$ conditionally approximates $T(t)$ on $N_{3}$ uniformly on compact sets of $\left[t_{0},+\infty\right)$.

Step 2. Estimate of $\left\|T_{h}(t) u_{0 h}-T(t) u_{0 h}\right\|_{V}$ for $t_{0} \leq t \leq t_{1}$ when $T_{h}(\tau) u_{0 h}$ and $T(\tau) u_{0 h}$ belong to $N_{3}$ for $0 \leq \tau \leq t$. To this end, we estimate the term $\left\|\tau T_{h}(\tau) u_{0 h}-\tau T(\tau) u_{0 h}\right\|_{V}$ for $0 \leq \tau \leq t$. We set $u(\tau)=T(\tau) u_{0 h}, u_{h}(\tau)=T_{h}(\tau) u_{0 h}$ 
$Z(\tau)=\tau u(\tau)$ and $Z_{h}(\tau)=\tau u_{h}(\tau)$. Using the equality $Q_{h} A=A_{h} P_{h}$, one easily shows that $Z_{h}(\tau)-P_{h} Z(\tau)$ satisfies the equation

$$
\begin{aligned}
& \frac{d}{d t}\left(Z_{h}-P_{h} Z\right)+A_{h}\left(Z_{h}-P_{h} Z\right) \\
& \quad=\tau Q_{h}\left(f\left(u_{h}\right)-f(u)\right)+\tau\left(\frac{d}{d t}\left(Q_{h} u-P_{h} u\right)\right)+u_{h}-P_{h} u .
\end{aligned}
$$

Taking the inner product in $H$ of (3.16) by $d\left(Z_{h}-P_{h} Z\right) / d t$, we obtain, thanks to the property (3.9)(ii),

$$
\begin{aligned}
&\left\|\frac{d}{d t}\left(Z_{h}-P_{h} Z\right)\right\|_{H}^{2}+a\left(Z_{h}-P_{h} Z, \frac{d}{d t}\left(Z_{h}-P_{h} Z\right)\right) \\
& \leq L\left\|Z_{h}-Z\right\|_{V}\left\|\frac{d}{d t}\left(Z_{h}-P_{h} Z\right)\right\|_{H} \\
&+\left\|\frac{d}{d t}\left(Z-P_{h} Z\right)\right\|_{H}\left\|\frac{d}{d t}\left(Z_{h}-P_{h} Z\right)\right\|_{H} \\
&+\left\|u_{h}-P_{h} u\right\|_{H}\left\|\frac{d}{d t}\left(Z_{h}-P_{h} Z\right)\right\|_{H} .
\end{aligned}
$$

Using the relation (3.12) (where $u_{h}-u_{0 h}$ is replaced by $Z_{h}-P_{h} Z$ ) and the hypothesis (3.2) as well as the inequality $a b \leq a^{2} / 2 \varepsilon+\varepsilon b^{2} / 2$, we derive from (3.17),

$$
\begin{aligned}
\frac{d}{d t} a\left(Z_{h}-P_{h} Z, Z_{h}-P_{h} Z\right) & \\
\leq & \left(L^{2}+C_{1}^{2}\right)\left\|Z_{h}-P_{h} Z\right\|_{V}^{2}+L^{2}\left\|Z-P_{h} Z\right\|_{V}^{2} \\
& +\left\|\frac{d}{d t}\left(Z-P_{h} Z\right)\right\|_{H}^{2}+\left\|u_{h}-P_{h} u\right\|_{H}^{2} .
\end{aligned}
$$

If we integrate (3.18) from 0 to $t$ and then apply Gronwall's inequality, we obtain

$$
\begin{aligned}
& \left\|\left(Z_{h}-P_{h} Z\right)(t)\right\|_{V}^{2} \\
& \leq C_{2} e^{C_{3} t}\left[\int _ { 0 } ^ { t } \left\{\left\|Z(s)-P_{h} Z(s)\right\|_{V}^{2}+\left\|u_{h}(s)-Q_{h} u(s)\right\|_{H}^{2}\right.\right. \\
& +\left\|u(s)-Q_{h} u(s)\right\|_{H}^{2}+\left\|u(s)-P_{h} u(s)\right\|_{H}^{2} \\
& \left.\left.+\left\|\frac{d}{d t}\left(Z(s)-P_{h} Z(s)\right)\right\|_{H}^{2}\right\} d s\right],
\end{aligned}
$$

where $C_{2}$ and $C_{3}$ are two positive constants independent of $h$ and $t$. We now estimate $\left\|u_{h}(\tau)-Q_{h} u(\tau)\right\|_{H}, 0 \leq \tau \leq t$. The function $Q_{h} u-u_{h}$ satisfies the equation

$$
\left\{\begin{array}{l}
\frac{d}{d t}\left(Q_{h} u-u_{h}\right)+A_{h}\left(Q_{h} u-u_{h}\right) \\
=Q_{h} f(u)-Q_{h} f\left(u_{h}\right)+\left(A_{h} Q_{h}-Q_{h} A\right) u \\
\left(Q_{h} u-u_{h}\right)(0)=0
\end{array}\right.
$$


Taking the inner product in $H$ of (3.20) by $Q_{h} u-u_{h}$ and using the property (3.9)(ii), we obtain

$$
\begin{aligned}
& \frac{1}{2} \frac{d}{d t}\left\|u_{h}-Q_{h} u\right\|_{H}^{2}+\gamma\left\|u_{h}-Q_{h} u\right\|_{V}^{2} \\
& \leq L\left\|u-Q_{h} u\right\|_{V}\left\|u_{h}-Q_{h} u\right\|_{H}+L\left\|u_{h}-Q_{h} u\right\|_{V}\left\|u_{h}-Q_{h} u\right\|_{H} \\
&+C_{0}\left\|u-Q_{h} u\right\|_{V}\left\|u_{h}-Q_{h} u\right\|_{V} .
\end{aligned}
$$

By Gronwall's inequality, the above estimate implies, after an integration from 0 to $\tau$,

$$
\left\|u_{h}(\tau)-Q_{h} u(\tau)\right\|_{H}^{2} \leq C_{4} e^{C_{5} \tau}\left(\int_{0}^{\tau}\left\|u(s)-Q_{h} u(s)\right\|_{V}^{2} d s\right)
$$

where $C_{4}$ and $C_{5}$ are two positive constants independent of $\tau$ and $h$. Thanks to the properties (3.6)(i) and (3.6)(ii), we deduce from (3.19) and (3.21), for $0 \leq t \leq t_{1}$,

$$
\begin{array}{r}
\left\|Z(t)-Z_{h}(t)\right\|_{V}^{2} \\
\leq C_{6} e^{C_{7} t} h^{2 m}\left\{\|t A u(t)\|_{H}^{2}+\int_{0}^{t}\left(\|A u(s)\|_{H}^{2}+\left\|s \frac{d u}{d t}(s)\right\|_{V}^{2}\right.\right. \\
\left.\left.+\|u(s)\|_{V}^{2}\right) d s\right\}
\end{array}
$$

where $C_{6}$ and $C_{7}$ are two positive constants independent of $t$ and $h$. On the one hand, using Henry [17, p. 71], one easily proves that, for $0<\tau \leq t$,

$$
\left\|\frac{d u}{d t}(\tau)\right\|_{V} \leq K_{0}\left(N_{2}, \delta_{0}\right) e^{K_{1} t_{1}} \frac{1}{\tau}
$$

where $K_{0}\left(N_{2}, \delta_{0}\right)$ is a positive constant depending only on $N_{2}$ and $\delta_{0}$, and $K_{1}$ is a positive constant.

Since $\tau A u=\tau f(u)-\tau d u / d t$, we infer from (3.23), for $0<\tau \leq t$,

$$
\|\tau A u(\tau)\|_{H} \leq t_{1} \sup _{v \in N_{3}}\|f(v)\|_{H}+K_{0}\left(N_{2}, \delta_{0}\right) e^{K_{1} t_{1}} .
$$

On the other hand, since $d u(\tau) / d t$ belongs to $H$ for $\tau>0$, we may consider the inner product in $H$ of Eq. (3.3) by $d u / d t$; thus, using a relation similar to (3.12), we obtain, for $0 \leq t \leq t_{1}$,

$$
\int_{0}^{t}\left\|\frac{d u}{d t}(s)\right\|_{H}^{2} d s \leq 2 \int_{0}^{t}\|f(u(s))\|_{H}^{2} d s+2 C_{1}^{2} \int_{0}^{t}\|u(s)\|_{V}^{2} d s+C_{0}\|u(t)\|_{V}^{2}
$$

Since $A u=f(u)-d u / d t$, we deduce from (3.25) that, for $0 \leq t \leq t_{1}$,

$$
\int_{0}^{t}\|A u(s)\|_{H}^{2} d s \leq K_{2}\left(N_{2}, \delta_{0}\right) e^{K_{3} t_{1}}
$$

where $K_{2}\left(N_{2}, \delta_{0}\right)$ is a positive constant depending only on $N_{2}$ and $\delta_{0}$, and $K_{3}$ is a positive constant.

Finally, the estimates (3.22), (3.23), (3.24) and (3.26) give us, for $0 \leq t \leq t_{1}$,

$$
\left\|Z(t)-Z_{h}(t)\right\|_{V} \leq K_{4}\left(N_{2}, \delta_{0}\right) e^{K_{5} t_{1}} h^{m}
$$

or, for $t_{0} \leq t \leq t_{1}$,

$$
\left\|u(t)-u_{h}(t)\right\|_{V} \leq K_{4}\left(N_{2}, \delta_{0}\right) \frac{e^{K_{5} t_{1}} h^{m}}{t_{0}}
$$


where $K_{4}\left(N_{2}, \delta_{0}\right)$ is a positive constant depending only on $N_{2}$ and $\delta_{0}$, and $K_{5}$ is a positive constant.

Remark 3.3. We also could have used the methods of Bramble, Schatz, Thomée and Wahlbin [3], Fujita and Mizutani [10], Helfrich [16] or Johnson, Larsson, Thomée and Wahlbin [20] for estimating $\left\|u(t)-u_{h}(t)\right\|_{V}$. For the estimate of $\left\|u(t)-u_{h}(t)\right\|_{V}$ when $u$ is more regular, we refer the reader to Thomée [33] and to Thomée and Wahlbin [34]. (See also the references in [33]).

Remark 3.4. Let $\Omega$ be a regular or convex, bounded domain in $\mathbf{R}^{n}, n=1,2,3$, and let $f: \mathbf{R} \rightarrow \mathbf{R}$ be a locally Lipschitz continuous function. Then, if $n=1$, the mapping $f: u \in H^{1}(\Omega) \rightarrow f(u(x)) \in L^{2}(\Omega)$ is also locally Lipschitz continuous. If, in the cases $n=2$ or $3, f$ satisfies the additional condition

$$
\forall v, \forall w \in \mathbf{R}, \quad|f(v)-f(w)| \leq C(1+|v|+|w|)^{\sigma}|v-w|
$$

where

$$
\sigma \leq \frac{2}{n-2} \quad \text { for } n \geq 3, \sigma \text { arbitrary for } n=2,
$$

then the mapping $f: u \in H^{1}(\Omega) \rightarrow f(u) \in L^{2}(\Omega)$ is also locally Lipschitz continuous. If the condition (3.28) is not satisfied, we have in general to work in a space other than $H^{1}(\Omega)$ (see Subsection 3.2 and Example 3.2 below).

3.2. An Extension of the Previous Result. Let us again consider the operator $A$ introduced in Subsection 3.1 that satisfies the properties (3.1) and (3.2). Now we assume that $f: V \rightarrow H$ is no longer Lipschitz continuous. But instead, we suppose that $A$ is a sectorial operator on a Banach space $Y \subset H$ and that $f: Y^{\alpha} \rightarrow Y$ is locally Lipschitz continuous, for a real number $\alpha, \frac{1}{2} \leq \alpha<1$. Furthermore, we assume that the following continuous inclusions hold:

$$
D_{Y}(A) \hookrightarrow Y^{\alpha} \hookrightarrow V \hookrightarrow Y \hookrightarrow H,
$$

where $D_{Y}(A)=\{y \in Y: A y \in Y\}$ and $Y^{\alpha}=D_{Y}\left(A^{\alpha}\right)$.

We assume that all the solutions $u\left(t, u_{0}\right)$ of (3.3) are defined and belong to $Y^{\alpha}$ for $t \geq 0$, if $u_{0} \in Y^{\alpha}$. Thus, the map $T_{Y}(t): Y^{\alpha} \rightarrow Y^{\alpha}, t \geq 0$, defined by $T_{Y}(t) u_{0}=u\left(t, u_{0}\right)$, becomes a $C^{0}$-semigroup on $Y^{\alpha}$. Finally we suppose that $T_{Y}(t)$ admits a compact attractor $A$ which attracts a bounded open set $O \supset A$. Then there exists an open neighborhood $\tilde{N}_{1}$ of $A$ such that $\tilde{N}_{1} \subset \mathcal{O}$ and $T_{Y}(t) \tilde{N}_{1} \subset \tilde{N}_{1}$ for $t \geq 0$.

Now we introduce a function $\tilde{f}$ which is globally Lipschitz continuous from $V$ into $H$ and coincides with $f$ on 0 . We consider the equation

$$
\left\{\begin{array}{l}
d \tilde{u} / d t+A \tilde{u}=\tilde{f}(\tilde{u}), \\
\tilde{u}(0)=u_{0} .
\end{array}\right.
$$

Obviously, if $u_{0} \in \tilde{N}_{1}$ then $\tilde{u}\left(t, u_{0}\right)=u\left(t, u_{0}\right)$ for $t \geq 0$. Let $\left(V_{h}\right)_{h}$ be the family of finite-dimensional subspaces of $V$ introduced in Subsection 3.1. We suppose that the spaces $V_{h}$ are included in $Y^{\alpha}$, satisfy the conditions (3.6) and the two following assumptions:

for any $\beta, \alpha<\beta \leq 1$, there exists a constant $\theta(\alpha, \beta)>0$ such that, for $v$ in $Y^{\beta}$,

$$
\left\|v-P_{h} v\right\|_{Y^{\alpha}} \leq C h^{2 m \theta(\alpha, \beta)}\|v\|_{Y^{\beta}}
$$


and

there exists a constant $\theta_{\alpha}, 0<\theta_{\alpha}<\frac{1}{2}$, such that, for any $v_{h}$ in $V_{h}$,

$$
\left\|v_{h}\right\|_{Y^{\alpha}} \leq C h^{-2 m \theta_{\alpha}}\left\|v_{h}\right\|_{V}
$$

We point out that, as in (3.6), the quantity $2 m$ essentially depends on the order of the differential operator $A$. We consider the approximate problem

$$
\left\{\begin{array}{l}
d \tilde{u}_{h} / d t+A_{h} \tilde{u}_{h}=Q_{h} \tilde{f}\left(\tilde{u}_{h}\right), \\
\tilde{u}_{h}(0)=u_{0 h},
\end{array}\right.
$$

for $u_{0 h} \in V_{h}$. We introduce the map $\tilde{T}_{h}(t): V_{h} \rightarrow V_{h}$ given by $\tilde{T}_{h}(t) u_{0 h}=$ $\tilde{u}_{h}\left(t, u_{0 h}\right)$. Since $\tilde{f}$ is globally Lipschitz continuous, $\tilde{T}_{h}(t) u_{0 h}$ exists for any $t \geq 0$.

THEOREM 3.2. Under the above hypotheses, there exists $h_{0}>0$ such that, for $h \leq h_{0}, \tilde{T}_{h}(t)$ admits a compact attractor $\tilde{A}_{h}$ which attracts the open set $\tilde{N}_{1} \cap V_{h}$ (where $\tilde{N}_{1}$ is given above). Moreover, $\delta_{Y^{\alpha}}\left(\tilde{A}_{h}, A\right) \rightarrow 0$ as $h \rightarrow 0$.

Proof. Let $t_{0}>0$ be a fixed real number. For any $t_{1}>t_{0}$, we are going to estimate $\left\|T_{Y}(t) u_{0 h}-\tilde{T}_{h}(t) u_{0 h}\right\|_{Y^{\alpha}}$ for $t_{0} \leq t \leq t_{1}$, when $u_{0 h} \in \tilde{N}_{1}$. We set $u(t)=T_{Y}(t) u_{0 h}, \tilde{u}_{h}(t)=\tilde{T}_{h}(t) u_{0 h}$. Recall that $u(t)=\tilde{u}(t)$. By virtue of the conditions (3.31), we have

$$
\begin{aligned}
\left\|u(t)-\tilde{u}_{h}(t)\right\|_{Y^{\alpha}} & \leq\left\|\tilde{u}(t)-P_{h} \tilde{u}(t)\right\|_{Y^{\alpha}}+\left\|P_{h} \tilde{u}(t)-\tilde{u}_{h}(t)\right\|_{Y^{\alpha}} \\
& \leq C h^{2 m \theta(\alpha, \beta)}\|u(t)\|_{Y^{\beta}}+C h^{-2 m \theta_{\alpha}}\left\|P_{h} \tilde{u}(t)-\tilde{u}_{h}(t)\right\|_{V}
\end{aligned}
$$

where $\alpha<\beta<1$.

Arguing as in Subsection 3.1 (see estimate (3.27)), we obtain

$$
h^{-2 m \theta_{\alpha}}\left\|P_{h} \tilde{u}(t)-\tilde{u}_{h}(t)\right\|_{V} \leq K_{4}\left(\tilde{N}_{1}\right) \frac{e^{K_{5} t_{1}}}{t_{0}} h^{2 m\left(1 / 2-\theta_{\alpha}\right)} .
$$

Finally, by using Henry [17, p. 57], we deduce from the above estimates, for $t_{0} \leq$ $t \leq t_{1}$,

$$
\left\|u(t)-\tilde{u}_{h}(t)\right\|_{Y^{\alpha}} \leq K_{6}\left(\beta, \tilde{N}_{1}\right) \frac{e^{K_{7} t_{1}}}{t_{0}} \sup \left(h^{2 m\left(1 / 2-\theta_{\alpha}\right)}, h^{2 m \theta(\alpha, \beta)}\right) .
$$

Since $\theta_{\alpha}<\frac{1}{2},(3.33)$ implies that the hypotheses of Proposition 2.1 hold and Theorem 3.2 is proved.

Example 3.2. Consider the equation

$$
\left\{\begin{array}{l}
d u / d t-\Delta u=f(u) \\
\left.u\right|_{\partial \Omega}=0 \\
u(t)_{/ t=0}=u_{0}
\end{array}\right.
$$

where, for instance, $\Omega$ is a convex polygonal domain in $\mathbf{R}^{2}$. If the function $f: \mathbf{R} \rightarrow$ $\mathbf{R}$ is locally Lipschitz continuous, but does not satisfy the condition (3.28), we cannot work in the space $V=H_{0}^{1}(\Omega)$. The map $f: w \in Y^{\alpha} \rightarrow f(w) \in Y$ is locally Lipschitz continuous if $Y=L^{2}(\Omega)$ and $\alpha>\frac{1}{2}$, or, if $Y=L^{p}(\Omega), p>2$ and $\alpha \geq \frac{1}{2}$. (Indeed in both cases, $Y^{\alpha} \hookrightarrow L^{\infty}(\Omega)$.)

Now assume that (3.34) admits an attractor $A$ in $Y^{\alpha}$ which attracts a bounded set $O \supset A$. So we can introduce the quantity

$$
B_{1}=\max _{v \in O}\|v\|_{L^{\infty}(\Omega)} \text {. }
$$


One easily constructs a function $\tilde{f}$ satisfying

$$
\tilde{f}(x)= \begin{cases}f(x) & \text { for }|x| \leq B_{1}, \\ 0 & \text { for }|x| \geq 2 B_{1} .\end{cases}
$$

The map $\tilde{f}: \quad w \in V \rightarrow \tilde{f}(w) \in H$ is globally Lipschitz continuous and coincides with $f$ on 0 .

Let us give an example of spaces $V_{h}$ in the case $Y^{\alpha}=H^{2 \alpha}(\Omega) \cap H_{0}^{1}(\Omega), \frac{1}{2}<\alpha<$ 1. Let $\left(\tau_{h}\right)_{h}$ be a uniformly regular family of triangulations in the sense of [5]. We set

$$
V_{h}=\left\{v_{h} \in C^{1}(\bar{\Omega}) \cap H_{0}^{1}(\Omega):\left.v_{h}\right|_{K} \in P_{3}(K), \forall K \in \tau_{h}\right\},
$$

where $P_{3}(K)$ is the space of all polynomials of degree $\leq 3$ on $K$. Then, of course, the hypotheses (3.6) are satisfied with $m=1$. Conditions (3.31)(i) and (3.31)(ii) hold with $\theta(\alpha, \beta)=\beta-\alpha$ and $\theta_{\alpha}=\alpha-\frac{1}{2}$.

3.3. Approximation of Sectorial Evolutionary Equations with Special Projection Methods. More generally, let $A$ be a sectorial linear operator on a Banach space $X$, and consider the nonlinear equation (3.3) where now we assume that there exists a real number $\alpha \in[0,1)$ such that $f: X^{\alpha} \rightarrow X$ is locally Lipschitz continuous and $u_{0} \in X^{\alpha}$. As in Subsection 3.1, we introduce the map $T(t): X^{\alpha} \rightarrow X^{\alpha}, t \geq 0$, defined by $T(t) u_{0}=u\left(t, u_{0}\right)$ and suppose that $T(t)$ has a (local) compact attractor $A$ which attracts an open set $O \supset A$. We assume that $\operatorname{Re} \sigma(A)>\gamma>0$, where $\sigma(A)$ denotes the spectrum of $A$ (if not, we replace $A$ by $A_{1}=A+a I, \operatorname{Re} \sigma\left(A_{1}\right)>\gamma$ and replace Eq. (3.3) by Eq. $\left.(3.3)^{\prime}\right)$. We also suppose that $\sigma(A)$ consists of isolated points $\lambda_{n}$ only, with no accumulation in the finite part of $\mathbf{C}$, and that each $\lambda_{n}$ is of finite order. We arrange the points $\lambda_{n}$ in such a way that

$$
\gamma<\operatorname{Re} \lambda_{1} \leq \operatorname{Re} \lambda_{2} \leq \cdots \leq \operatorname{Re} \lambda_{n} \leq \operatorname{Re} \lambda_{n+1} \leq \cdots,
$$

where $\operatorname{Re} \lambda_{n} \rightarrow+\infty$ as $n \rightarrow+\infty$.

We denote by $\Phi_{n}$ the generalized eigenspace corresponding to $\lambda_{n}$, by $P_{N}$ the projection from $X$ onto the space $\left[\Phi_{1}, \Phi_{2}, \ldots, \Phi_{N}\right]$ and by $Q_{N}$ the projection $I-P_{N}$. We assume that, for $0 \leq \beta<1,\left\|P_{N}\right\|_{\mathcal{L}\left(X^{\beta} ; X^{\beta}\right)}$ is bounded by a constant $K_{\beta}>0$, uniformly with respect to $N$. By $[17$, p. 21], for any $\varepsilon>0$, for any integer $N$, there exists a constant $K_{\varepsilon, N}$ such that

$$
\left\|A^{j} e^{-A t} Q_{N}\right\|_{\mathcal{L}(X, X)} \leq K_{\varepsilon, N} \frac{e^{-\left(\operatorname{Re} \lambda_{N+1}-\varepsilon\right) t}}{t^{j}} \quad \text { for } j=0,1 .
$$

Below, we assume that, for $0 \leq \beta<1$,

$$
\lim _{N \rightarrow+\infty} \frac{K_{\varepsilon, N}}{\left(\operatorname{Re} \lambda_{N+1}-\varepsilon\right)^{\beta}}=0
$$

this condition being usually satisfied.

Now let us consider the following equation on $X_{N}=P_{N} X$ :

$$
\left\{\begin{array}{l}
d u_{N} / d t+A u_{N}=P_{N} f\left(u_{N}\right), \\
u_{N}(0)=u_{0 N}
\end{array}\right.
$$

where $u_{0 N} \in X_{N}$. Equation (3.3) $)_{N}$ is an ordinary differential equation. Let us introduce the map $T_{N}(t): X_{N} \rightarrow X_{N}$, defined by $T_{N}(t) u_{0 N}=u_{N}\left(t, u_{0 N}\right)$, as long as $u_{N}\left(t, u_{0 N}\right)$ exists. 
THEOREM 3.3. Under the above hypotheses, there exists a number $N_{0}>0$ such that, for $N \geq N_{0}, T_{N}(t)$ admits a local compact attractor $\mathcal{A}_{N}$ which attracts an open set $O_{1} \cap X_{N}$, where $O_{1}$ is independent of $N$. Moreover, $\delta_{X^{\alpha}}\left(\mathcal{A}_{N}, \mathcal{A}\right) \rightarrow 0$ as $N \rightarrow+\infty$.

The proof of this theorem is very easy and is a consequence of Proposition 2.2 (the complete proof can be found in Hale, Lin and Raugel [15]).

4. Semidiscretization in Time of Some Parabolic Problems. We keep the same notations and the same assumptions as in Subsection 3.1, but here we moreover assume that the operator $A$ is selfadjoint and has a compact resolvent. (The generalization of the following results to the case where $A$ is not selfadjoint, but satisfies the condition (3.2), is left to the reader.) As in Subsection 3.1, we assume that $\gamma_{0}=0$, and we consider the nonlinear equation

$$
\left\{\begin{array}{l}
d u / d t+A u=f(u) \\
u(0)=u^{0}
\end{array}\right.
$$

where $u^{0} \in V$ and $f \in C^{2}(V ; H)$, for instance. The hypotheses on $f$ can be weakened. Now let us turn to a semidiscretization in time of Eq. (4.1) by a onestep method. More precisely, let $k$ be a positive time increment, let $t_{n}=n k, n \geq 0$, and define an approximation $u_{n}$ of the solution $u$ of (4.1) at time $t_{n}$ by the recursion formula

$$
\left\{\begin{array}{l}
u_{n+1}=(1-(1-\theta) k A)(1+\theta k A)^{-1} u_{n}+k(1+\theta k A)^{-1} f\left(u_{n}\right), \\
u_{0}=u^{0},
\end{array}\right.
$$

where $\frac{1}{2}<\theta \leq 1$.

Remark 4.1. The results that we are going to prove below are also valid if we replace $f\left(u_{n}\right)$ in $(4.1)_{k}$ by $f\left(\theta u_{n+1}+(1-\theta) u_{n}\right)$. But then the "linearized" scheme $(4.1)_{k}$ becomes a nonlinear one.

More generally, the following results are also true if we replace $(4.1)_{k}$ by a scheme that is strictly accurate of order 1 in the sense of Brenner, Crouzeix, Thomée [4] and is of the form

$$
\left\{\begin{array}{l}
u_{n+1}=r(k A) u_{n}+k \sum_{j=1}^{m} q_{j}(k A) f\left(u_{n}\right), \\
u_{0}=u^{0},
\end{array}\right.
$$

where $r, q_{1}, \ldots, q_{m}$ are rational functions of the variable $z$ which are bounded, as well as $z q_{j}(z), 1 \leq j \leq m$, for $z \geq 0$, and where $|r(z)|<1$, for $z \geq 0$, and $|r(\infty)| \neq 1$. The proof, in the case of the scheme $(4.2)_{k}$, uses the same arguments as below and the property that $r(z)$ can be written as $(1-z s(z)) /(1+\sigma z)$, where $\sigma$ is a suitable positive constant (for more details, see Raugel [29]).

Now we introduce the mapping $T_{k} \in \mathcal{L}(V, V)$ defined by $T_{k} u^{0}=u_{1}$, where $u_{1}$ is given by the formula (4.1) $)_{k}$. For any integer $n \geq 1, T_{k}^{n} u^{0}=u_{n}$. We remark that $T_{k}$ is well defined on the whole space $V$ and that $T_{k}^{n}: \mathbf{N} \rightarrow C^{0}(V, V)$ is a discrete semigroup. Although Sections 1 and 2 deal with $C^{0}$-semigroups $T(t): \mathbf{R}^{+} \rightarrow$ $C^{0}(V ; V)$ only, the definitions and the results contained there obviously extend to discrete semigroups. For instance, a set $B \subset V$ is said to attract a set $C \subset V$ under $T_{k}$ if, for any $\varepsilon>0$, there is an integer $n_{0}=n_{0}(B, C, \varepsilon)$ such that $T_{k}^{n} C \subset \mathcal{N}(B, \varepsilon)$ 
for $n \geq n_{0}$ (the definitions of a local attractor and an attractor are unchanged; for more details, see Hale [11], for instance).

Here we suppose that the map $T(t): V \rightarrow V, t \geq 0$, defined by $T(t) u^{0}=u(t)$, where $u(t)$ is the solution of (4.1), admits a local, compact attractor $A$ which attracts a bounded open set $0,0 \supset A$.

THEOREM 4.1. Under the above hypotheses, there exists $k_{0}>0$, such that, for $k \leq k_{0}$, the process $T_{k}$ admits a local, compact attractor $A_{k}$, which attracts an open set $N_{1}$, where $N_{1}$ is independent of $k, N_{1} \supset A_{k}$ for every $k$. Moreover, $\delta_{V}\left(A_{k}, A\right) \rightarrow 0$ as $k \rightarrow 0$.

The remainder of this section will be devoted to the proof of Theorem 4.1. But, beforehand, let us recall the following discrete analogue of Gronwall's lemma, the proof of which is left to the reader.

LEMMA 4.2. Let $\left(a_{n}\right)_{n},\left(b_{n}\right)_{n},\left(c_{n}\right)_{n}$ be three sequences of positive real numbers such that $\left(c_{n}\right)_{n}$ is monotonically increasing and

$$
a_{n}+b_{n} \leq c_{n}+\lambda \sum_{m=0}^{n-1} a_{m} \quad \text { for } n \geq 1 \text { and } \lambda>0
$$

with $a_{0}+b_{0} \leq c_{0}$. Then, these sequences also satisfy

$$
a_{n}+b_{n} \leq c_{n} \exp (\lambda n) \quad \text { for } n \geq 0 .
$$

Only for the sake of simplicity, we consider that the space $V$ is equipped with the norm

$$
\forall v \in V, \quad\|v\|_{V}=(A v, v)^{1 / 2}
$$

Hence, the dual norm on $V^{\prime}$ is given by

$$
\forall v^{\prime} \in V^{\prime}, \quad\left\|v^{\prime}\right\|_{V^{\prime}}=\left(A^{-1} v^{\prime}, v^{\prime}\right)^{1 / 2} .
$$

Proof of Theorem 4.1. In order to prove Theorem 4.1, we shall apply the following modified version of Theorem 2.4, the proof of which is left to the reader. Clearly, the conclusions of Theorem 2.4 and hence of Theorem 4.1 hold, if the following conditions are satisfied:

There exist four positive constants $k_{0}, \delta_{0}, \delta_{1}, \alpha_{0}$, with $\alpha_{0}>k_{0}$, and two open neighborhoods $\mathcal{N}_{1}, \mathcal{N}_{2}$ of $A$, with $\mathcal{N}_{1} \subset \mathcal{N}_{2}$, such that, for $0<k \leq k_{0}$,

(i) $T_{k}$ is an asymptotically smooth map (this condition holds in particular if $T_{k}=T_{1 k}+T_{2 k}$, where $T_{1 k}$ is completely continuous and $T_{2 k}$ is a linear strict contraction);

(ii) $T(t) \mathcal{N}_{1} \subset \mathcal{N}_{2}$ for $t \geq 0$,

(iii) $T_{k}^{n} \mathcal{N}_{1} \subset \mathcal{N}_{2}$ for $0 \leq n \leq \alpha_{0} / k$,

(iv) $T_{k} \mathcal{N}\left(\mathcal{N}_{2}, \delta_{0}\right) \subset \mathcal{N}_{3}$, where $\mathcal{N}_{3}=\mathcal{N}\left(\mathcal{N}_{2}, \delta_{0}+\delta_{1}\right)$; and

(v) for any $\alpha_{1}>\alpha_{0}$, there exist a constant $k_{0}\left(\alpha_{1}, \mathcal{N}_{3}\right)$ with $0<k_{0}\left(\alpha_{1}, \mathcal{N}_{3}\right)<k_{0}$, and a function $\eta\left(k, \alpha_{1}, \mathcal{N}_{3}\right)$ defined for $0<k \leq k_{0}\left(\alpha, \mathcal{N}_{3}\right)$ such that

$$
\lim _{k \rightarrow \infty} \eta\left(k, \alpha_{1}, \mathcal{N}_{3}\right)=0
$$


and, for any $0<k \leq k_{0}\left(\alpha_{1}, \mathcal{N}_{3}\right)$, if $u^{0} \in \mathcal{N}_{3}$ has the property that $T_{k}^{n} u^{0}$ and $T(n k) u^{0}$ belong to $\mathcal{N}_{3}$ for $0 \leq n \leq \alpha_{2} / k$ and $0 \leq n k \leq \alpha_{2}+k_{0}$, respectively (where $\left.\alpha_{0} \leq \alpha_{2} \leq \alpha_{1}\right)$, then

$$
\left\|T_{k}^{n} u^{0}-T(n k) u^{0}\right\|_{V} \leq \eta\left(k, \alpha_{1}, \mathcal{N}_{3}\right) \quad \text { for } \frac{\alpha_{0}}{k} \leq n \leq \frac{\alpha_{2}}{k} .
$$

Now we show in four steps that the above conditions are satisfied.

(1) By $(4.1)_{k}$, we can write, for any $u^{0} \in V$,

$$
\begin{aligned}
T_{k} u^{0} & =\left[(1+\theta k A)^{-1} u^{0}+k(1+\theta k A)^{-1} f\left(u^{0}\right)\right]-(1-\theta) k A(1+\theta k A)^{-1} u^{0} \\
& \equiv T_{1 k} u^{0}+T_{2 k} u^{0} .
\end{aligned}
$$

Let $B$ be a bounded set in $H$; for any $v \in B$, we have $\left\|k A(1+\theta k A)^{-1} v\right\|_{H} \leq\|v\|_{H}$. Hence, for any fixed positive $k,(1+\theta k A)^{-1} B$ is a bounded set in $D(A)$. Since $D(A) \subset V$ is a compact embedding, this proves that $T_{1 k}$ is completely continuous. On the other hand, as $A$ is an elliptic operator, $T_{2 k}$, for $k>0$, is a linear strict contraction as soon as $2 \theta-1>0$. Condition (i) is proved.

(2) As $A$ is a compact attractor, there is a bounded open neighborhood $N_{1}$ of $A$ such that $\mathcal{N}_{1} \subset 0$ and $T(t) \mathcal{N}_{1} \subset \mathcal{N}_{1}$ for $t \geq 0$. Let $B_{0}=\max _{v \in \mathcal{N}_{1}}\|v\|_{V}$ and $B_{1}=\max _{v \in \mathcal{N}_{1}}\|f(v)\|_{H}$; we set $\varepsilon_{0}=4\left(B_{0}^{2}+B_{1}^{2}\right)^{1 / 2}$ and $\mathcal{N}_{2}=\mathcal{N}\left(\mathcal{N}_{1}, \varepsilon_{0}\right)$. Finally, we choose a real number $\delta_{0}>0$ and we set $\delta_{1}=2\left[\left(B_{0}+\varepsilon_{0}+\delta_{0}\right)^{2}+B_{2}^{2}\right]^{1 / 2}$, where $B_{2}=\max _{v \in \mathcal{N}\left(\mathcal{N}_{2}, \delta_{0}\right)}\|f(v)\|_{H}$.

We remark that the condition (iii) is an immediate consequence of the following property:

$$
\left\{\begin{array}{l}
\text { There exists a constant } \alpha_{0}>0 \text { independent of } k \text { such that, for } \\
\text { any } u^{0} \in \mathcal{N}_{1}, \text { if } T_{k}^{n} u^{0} \text { belongs to } \mathcal{N}\left(u^{0}, \varepsilon_{0}\right), \text { for } 0 \leq n \leq \beta\left(k, u^{0}\right) / k \\
\text { with } 0 \leq \beta\left(k, u^{0}\right) \leq \alpha_{0}-k, \text { then } T_{k}^{n} u^{0} \text { belongs to } \mathcal{N}\left(u^{0}, \varepsilon_{0}\right) \text { for } \\
0 \leq n \leq\left(\beta\left(k, u^{0}\right) / k\right)+1 .
\end{array}\right.
$$

Let $u^{0} \in \mathcal{N}_{1}$. We set $u_{n}=T_{k}^{n} u^{0}, \tilde{u}_{n}=u_{n}-u^{0}$ and we assume that, for $0 \leq n \leq$ $\beta\left(k, u^{0}\right) / k, T_{k}^{n} u^{0} \in \mathcal{N}\left(u^{0}, \varepsilon_{0}\right)$. By $(4.1)_{k}$, we have

$$
\tilde{u}_{n}-\tilde{u}_{n-1}+k A\left(\theta \tilde{u}_{n}+(1-\theta) \tilde{u}_{n-1}\right)=k f\left(u_{n-1}\right)-k A u^{0} .
$$

Taking the inner product in $H$ of (4.8) by $\tilde{u}_{n}-\tilde{u}_{n-1}$, we obtain

$$
\begin{aligned}
\| \tilde{u}_{n}- & \tilde{u}_{n-1}\left\|_{H}^{2}+\frac{k}{2}\right\| \tilde{u}_{n}\left\|_{V}^{2}-\frac{k}{2}\right\| \tilde{u}_{n-1}\left\|_{V}^{2}+\frac{k}{2}(2 \theta-1)\right\| \tilde{u}_{n}-\tilde{u}_{n-1} \|_{V}^{2} \\
\leq & k\left(f\left(u_{n-1}\right)-f\left(u^{0}\right), \tilde{u}_{n}-\tilde{u}_{n-1}\right) \\
& +k\left(f\left(u^{0}\right), \tilde{u}_{n}-\tilde{u}_{n-1}\right)+k\left(A u^{0}, \tilde{u}_{n}-\tilde{u}_{n-1}\right),
\end{aligned}
$$

or also,

$$
\left\|\tilde{u}_{n}\right\|_{V}^{2}-\left\|\tilde{u}_{n-1}\right\|_{V}^{2} \leq k L^{2}\left\|\tilde{u}_{n-1}\right\|_{V}^{2}+k B_{1}^{2}+\left(A u^{0}, \tilde{u}_{n}-\tilde{u}_{n-1}\right),
$$

where $L>0$ is the Lipschitz constant of $f$ on $\mathcal{N}_{3}$.

Summation over $n$ yields

$$
\left\|\tilde{u}_{m+1}\right\|_{V}^{2} \leq k L^{2} \sum_{n=0}^{m}\left\|\tilde{u}_{n}\right\|_{V}^{2}+k(m+1) B_{1}^{2}+\left\|u^{0}\right\|_{V}\left\|\tilde{u}_{m+1}\right\|_{V}
$$

where $m$ is the integral part of $\beta\left(k, u^{0}\right) / k$. Using Lemma 4.2, we infer from (4.9),

$$
\left\|\tilde{u}_{m+1}\right\|_{V}^{2} \leq\left[B_{0}^{2}+2 k(m+1) B_{1}^{2}\right] \exp \left(2 k L^{2}(m+1)\right) .
$$


Let now $\alpha_{0}$ be a positive constant such that

$$
\left[B_{0}^{2}+2 \alpha_{0} B_{1}^{2}\right] \exp \left(2 L^{2} \alpha_{0}\right)<\varepsilon_{0}
$$

and choose $k_{0}$ such that $0<k_{0}<\alpha_{0}$. Then one deduces from (4.10) that $\tilde{u}_{m+1} \in$ $\mathcal{N}\left(u^{0}, \varepsilon_{0}\right)$ if $m+1 \leq \alpha_{0} / k$, for $0<k \leq k_{0}$. Thus, property (A) is shown. As the proof of the condition (iv) uses similar estimates, it is left to the reader.

(3) Some auxiliary estimates. We shall estimate

$$
k \sum_{n=0}^{m}\left\|T(n k) u^{0}-T_{k}^{n} u^{0}\right\|_{V}^{2}
$$

and

$$
\sum_{n=0}^{m}\left\|\left(T((n+1) k) u^{0}-T_{k}^{n+1} u^{0}\right)-\left(T(n k) u^{0}-T_{k}^{n} u^{0}\right)\right\|_{H}^{2}
$$

for $0 \leq m \leq \alpha_{1} / k$, when $T_{k}^{n} u^{0}$ and $T(n k) u^{0}$ belong to $\mathcal{N}_{3}$ for $0 \leq n \leq m$ and $0 \leq n k \leq m k+k_{0}$, respectively.

We set $t_{n}=n k$ and $e_{n} \equiv T_{k}^{n} u^{0}-T(n k) u^{0} \equiv u_{n}-u\left(t_{n}\right)$. As it was pointed out in Raugel [29, proof of Theorem 2.2], one easily shows that

$$
\begin{aligned}
& k \sum_{n=0}^{m}\left\|e_{n}\right\|_{V}^{2}+\theta k\left\|e_{m+1}\right\|_{V}^{2}-k \sum_{n=0}^{m} \theta(1-\theta)\left\|e_{n+1}-e_{n}\right\|_{V}^{2} \\
& \quad \leq k \sum_{n=0}^{m}\left\|\theta e_{n+1}+(1-\theta) e_{n}\right\|_{V}^{2} .
\end{aligned}
$$

From the equations (4.1) and $(4.1)_{k}$ we infer

$$
\begin{aligned}
e_{n+1}- & e_{n}+k A\left(\theta e_{n+1}+(1-\theta) e_{n}\right) \\
= & k\left(f\left(u_{n}\right)-f\left(u\left(t_{n}\right)\right)\right)-\int_{t_{n}}^{t_{n+1}}\left(\frac{d u}{d t}(s)-\frac{d u}{d t}\left(t_{n}\right)\right) d s \\
& +\theta k A\left(u\left(t_{n}\right)-u\left(t_{n+1}\right)\right) .
\end{aligned}
$$

Taking the inner product in $H$ of (4.13) by $\theta e_{n+1}+(1-\theta) e_{n}+\gamma_{1}\left(e_{n+1}-e_{n}\right)$, where $\gamma_{1}>0$, we obtain the following inequality:

$$
\begin{aligned}
& \frac{1}{2}\left\|e_{n+1}\right\|_{H}^{2}-\frac{1}{2}\left\|e_{n}\right\|_{H}^{2}+\frac{1}{2}(2 \theta-1)\left\|e_{n+1}-e_{n}\right\|_{H}^{2}+k\left\|\theta e_{n+1}+(1-\theta) e_{n}\right\|_{V}^{2} \\
& \quad+\gamma_{1}\left\|e_{n+1}-e_{n}\right\|_{H}^{2}+\frac{k \gamma_{1}}{2}\left\|e_{n+1}\right\|_{V}^{2}-\frac{k \gamma_{1}}{2}\left\|e_{n}\right\|_{V}^{2}+\frac{k \gamma_{1}}{2}(2 \theta-1)\left\|e_{n+1}-e_{n}\right\|_{V}^{2} \\
& \leq k L\left\|e_{n}\right\|_{V}\left[\left(\theta+\gamma_{1}\right)\left\|e_{n+1}-e_{n}\right\|_{H}+\left\|e_{n}\right\|_{H}\right] \\
& \quad+\theta k\left\|u\left(t_{n}\right)-u\left(t_{n+1}\right)\right\|_{V}\left[\left\|\theta e_{n+1}+(1-\theta) e_{n}\right\|_{V}+\gamma_{1}\left\|e_{n+1}-e_{n}\right\|_{V}\right] \\
& \quad+\left\|\int_{t_{n}}^{t_{n+1}}\left(\frac{d u}{d t}(s)-\frac{d u}{d t}\left(t_{n}\right)\right) d s\right\|_{V^{\prime}}\left[\left\|\theta e_{n+1}+(1-\theta) e_{n}\right\|_{V}+\gamma_{1}\left\|e_{n+1}-e_{n}\right\|_{V}\right] .
\end{aligned}
$$


Using the inequality $a b \leq a^{2} / 2 \varepsilon+\varepsilon b^{2} / 2$ several times, we derive from the above estimate:

$$
\begin{aligned}
&\left\|e_{n+1}\right\|_{H}^{2}-\left\|e_{n}\right\|_{H}^{2}+k\left\|\theta e_{n+1}+(1-\theta) e_{n}\right\|_{V}^{2}+\gamma_{1}\left\|e_{n+1}-e_{n}\right\|_{H}^{2} \\
&+k \gamma_{1}\left\|e_{n+1}\right\|_{V}^{2}-k \gamma_{1}\left\|e_{n}\right\|_{V}^{2}+\frac{k \gamma_{1}}{2}(2 \theta-1)\left\|e_{n+1}-e_{n}\right\|_{V}^{2} \\
& \leq\left(\frac{k^{2} \theta^{2} L^{2}}{2 \theta-1}+\frac{\gamma_{1} k^{2} L^{2}}{2}+k \varepsilon_{0}\right)\left\|e_{n}\right\|_{V}^{2}+\frac{k L^{2}}{\varepsilon_{0}}\left\|e_{n}\right\|_{H}^{2} \\
&+k\left(2 \theta^{2}+\frac{4 \gamma_{1} \theta^{2}}{2 \theta-1}\right)\left\|u\left(t_{n}\right)-u\left(t_{n+1}\right)\right\|_{V}^{2} \\
&+\left(2+\frac{4 \gamma_{1}}{2 \theta-1}\right) \int_{t_{n}}^{t_{n+1}}\left\|\frac{d u}{d t}(s)-\frac{d u}{d t}\left(t_{n}\right)\right\|_{V^{\prime}}^{2} d s
\end{aligned}
$$

where $\varepsilon_{0}>0$ is a small enough constant.

Summation of (4.14) over $n$ yields

$$
\begin{aligned}
\left\|e_{m+1}\right\|_{H}^{2}+ & k \sum_{n=0}^{m}\left\|\theta e_{n+1}+(1-\theta) e_{n}\right\|_{V}^{2}+\gamma_{1} \sum_{n=0}^{m}\left\|e_{n+1}-e_{n}\right\|_{H}^{2} \\
& +k \gamma_{1}\left\|e_{m+1}\right\|_{V}^{2}+\frac{k \gamma_{1}}{2}(2 \theta-1) \sum_{n=0}^{m}\left\|e_{n+1}-e_{n}\right\|_{V}^{2} \\
\leq & k\left(\frac{k \theta^{2} L^{2}}{2 \theta-1}+\frac{\gamma_{1} k L^{2}}{2}+\varepsilon_{0}\right) \sum_{n=0}^{m}\left\|e_{n}\right\|_{V}^{2}+\frac{k L^{2}}{\varepsilon_{0}} \sum_{n=0}^{m}\left\|e_{n}\right\|_{H}^{2} \\
& +k\left(2 \theta^{2}+\frac{4 \gamma_{1} \theta^{2}}{2 \theta-1}\right) \sum_{n=0}^{m}\left\|u\left(t_{n}\right)-u\left(t_{n+1}\right)\right\|_{V}^{2} \\
& +\left(2+\frac{4 \gamma_{1}}{2 \theta-1}\right) \sum_{n=0}^{m} \int_{t_{n}}^{t_{n+1}}\left\|\frac{d u}{d t}(s)-\frac{d u}{d t}\left(t_{n}\right)\right\|_{V^{\prime}}^{2} d s .
\end{aligned}
$$

Now we set $\gamma_{1}=\sup (1,2 \theta(1-\theta) /(2 \theta-1))$ and we choose $k_{0}>0$ and $\varepsilon_{0}>0$ such that, for $0<k \leq k_{0}, k \theta^{2} L^{2} /(2 \theta-1)+\gamma_{1} k L^{2} / 2+\varepsilon_{0}<\frac{1}{2}$. Then, thanks to (4.12), we deduce from the previous inequality that

$$
\begin{aligned}
\left\|e_{m+1}\right\|_{H}^{2}+\frac{k}{2} \sum_{n=0}^{m}\left\|e_{n}\right\|_{V}^{2}+\sum_{n=0}^{m}\left\|e_{n+1}-e_{n}\right\|_{H}^{2} \\
\leq \frac{k L^{2}}{\varepsilon_{0}} \sum_{n=0}^{m}\left\|e_{n}\right\|_{H}^{2}+C(\theta) k \sum_{n=0}^{m}\left\|u\left(t_{n+1}\right)-u\left(t_{n}\right)\right\|_{V}^{2} \\
\quad+C(\theta) \sum_{n=0}^{m} \int_{t_{n}}^{t_{n+1}}\left\|\frac{d u}{d t}(s)-\frac{d u}{d t}\left(t_{n}\right)\right\|_{V^{\prime}}^{2} d s .
\end{aligned}
$$

Using Lemma 4.2 we infer from (4.15):

$$
\begin{aligned}
\left\|e_{m+1}\right\|_{H}^{2}+ & \frac{k}{2} \sum_{n=0}^{m}\left\|e_{n}\right\|_{V}^{2}+\sum_{n=0}^{m}\left\|e_{n+1}-e_{n}\right\|_{H}^{2} \\
(4.16) \leq & C(\theta) \exp \left(\frac{k L^{2}(m+1)}{\varepsilon_{0}}\right) \\
& \times\left[k \sum_{n=0}^{m}\left\|u\left(t_{n+1}\right)-u\left(t_{n}\right)\right\|_{V}^{2}+\sum_{n=0}^{m} \int_{t_{n}}^{t_{n+1}}\left\|\frac{d u}{d t}(s)-\frac{d u}{d t}\left(t_{n}\right)\right\|_{V^{\prime}}^{2} d s\right] .
\end{aligned}
$$


Let us set $B_{3}=\max _{v \in \mathcal{N}_{3}}\|v\|_{V}$. Then we can write

$$
\sum_{n=0}^{m}\left\|u\left(t_{n+1}\right)-u\left(t_{n}\right)\right\|_{V}^{2} \leq 2 B_{3}^{2}+k \int_{t_{1}}^{t_{m+1}}\left\|\frac{d u}{d t}\right\|_{V}^{2} d s .
$$

On the other hand, we have

$$
\begin{aligned}
& \sum_{n=0}^{m} \int_{t_{n}}^{t_{n+1}}\left\|\frac{d u}{d t}(s)-\frac{d u}{d t}\left(t_{n}\right)\right\|_{V^{\prime}}^{2} d s \\
& \quad \leq 2 k \sup _{t \in\left[0, t_{1}\right]}\left\|\frac{d u}{d t}(t)\right\|_{V^{\prime}}^{2}+k^{2} \int_{t_{1}}^{t_{m+1}}\left\|\frac{d^{2} u}{d t^{2}}\right\|_{V^{\prime}}^{2} d s
\end{aligned}
$$

and, as $A^{-1 / 2} d u / d t=A^{-1 / 2} f(u)-A^{1 / 2} u$,

$$
\sup _{0 \leq t \leq t_{m+1}}\left\|\frac{d u}{d t}(t)\right\|_{V^{\prime}}^{2} \leq B_{3}^{2}+\max _{v \in \mathcal{N}_{3}}\|f(v)\|_{H}^{2} .
$$

It now remains to bound the quantities

$$
\int_{t_{1}}^{t_{m+1}} k\left\|\frac{d u}{d t}\right\|_{V}^{2} d s \quad \text { and } \quad \int_{t_{1}}^{t_{m+1}} k\left\|\frac{d^{2} u}{d t^{2}}\right\|_{V^{\prime}}^{2} d s .
$$

By Henry [17, p. 71], there exist two constants $K_{0}>0$ and $K_{1}\left(\mathcal{N}_{3}\right)>0$ such that, for $0 \leq t \leq m k+k_{0}$,

$$
t\left\|\frac{d u}{d t}(t)\right\|_{V}+t^{1 / 2}\left\|\frac{d u}{d t}(t)\right\|_{H} \leq K_{1}\left(\mathcal{N}_{3}\right) e^{K_{0}\left(\alpha_{1}+k_{0}\right)} .
$$

Since the inequalities (3.25) and (4.20) hold, $f^{\prime}(u) t d u / d t+d u / d t$ belongs to $L^{2}\left(\left[0, t_{m+1}\right] ; H\right)$ and one easily proves that the function $t d u / d t$ satisfies the equation

$$
\left\{\begin{array}{l}
\text { (i) }\left(\frac{d}{d t}\left(t \frac{d u}{d t}\right), \phi\right)+a\left(t \frac{d u}{d t}, \phi\right)=\left(f^{\prime}(u) t \frac{d u}{d t}, \phi\right)+\underset{\left(\frac{d u}{d t}, \phi\right)}{\text { for } \phi \in V ;} \\
\text { (ii) }\left(t \frac{d u}{d t}\right)_{/ t=0}=0 .
\end{array}\right.
$$

Hence, $t d u / d t$ belongs to the space $H^{1}\left(\left[0, t_{m+1}\right] ; H\right)$. For $t>0$, Eq. (4.21)(i) can also be written as

$$
\left(t \frac{d^{2} u}{d t^{2}}, \phi\right)+a\left(t \frac{d u}{d t}, \phi\right)=\left(f^{\prime}(u) t \frac{d u}{d t}, \phi\right) \quad \text { for any } \phi \in V \text {. }
$$

We set $\phi=d u / d t$ in (4.22); then, after an integration from 0 to $t_{m+1}$, we obtain

$$
\begin{aligned}
\frac{1}{2}(t & \left.\left\|\frac{d u}{d t}\right\|_{H}^{2}\right)\left(t_{m+1}\right)+\gamma \int_{0}^{t_{m+1}} t\left\|\frac{d u}{d t}\right\|_{V}^{2} d t \\
\leq & \frac{1}{2} \int_{0}^{t_{m+1}}\left\|f^{\prime}(u) t \frac{d u}{d t}\right\|_{H}^{2} d t+\frac{3}{2} \int_{0}^{t_{m+1}}\left\|\frac{d u}{d t}\right\|_{H}^{2} d t \\
& +\left(\frac{1}{2} t\left\|\frac{d u}{d t}\right\|_{H}^{2}\right)(0) .
\end{aligned}
$$


Since

$$
\int_{0}^{t_{m+1}}\left\|f^{\prime}(u) t \frac{d u}{d t}\right\|_{H}^{2} d t \leq \sup _{u \in \mathcal{N}_{3}}\left\|f^{\prime}(u)\right\|_{\mathcal{L}(V ; H)}^{2} \int_{0}^{t_{m+1}} t^{2}\left\|\frac{d u}{d t}\right\|_{V}^{2} d t,
$$

we deduce from (4.23), by using (3.25) and (4.20), that

$$
\int_{0}^{t_{m+1}} t\left\|\frac{d u}{d t}\right\|_{V}^{2} d t \leq K_{2}\left(\mathcal{N}_{3}\right) e^{K_{3}\left(\alpha_{1}+k_{0}\right)}
$$

where $K_{2}\left(\mathcal{N}_{3}\right)$ and $K_{3}$ are two positive constants.

If we set $\phi=A^{-1} d^{2} u / d t^{2}$ in (4.22), we obtain

$$
\begin{aligned}
\frac{1}{2} \int_{0}^{t_{m+1}} t\left\|A^{-1 / 2} \frac{d^{2} u}{d t^{2}}\right\|_{H}^{2} d t+\frac{1}{2}\left(t\left\|\frac{d u}{d t}\right\|_{H}^{2}\right)\left(t_{m+1}\right) \\
\leq \frac{1}{2} \int_{0}^{t_{m+1}}\left\|A^{-1 / 2} f^{\prime}(u) t^{1 / 2} \frac{d u}{d t}\right\|_{H}^{2} d t+\int_{0}^{t_{m+1}}\left\|\frac{d u}{d t}\right\|_{H}^{2} d t \\
\quad+\frac{1}{2}\left(t\left\|\frac{d u}{d t}\right\|_{H}^{2}\right)(0),
\end{aligned}
$$

which implies, thanks to (3.25), (4.20) and (4.24), that

$$
\int_{0}^{t_{m+1}} t\left\|\frac{d^{2} u}{d t^{2}}(t)\right\|_{V^{\prime}}^{2} d t \leq K_{4}\left(\mathcal{N}_{3}\right) e^{K_{5}\left(\alpha_{1}+k_{0}\right)}
$$

where $K_{4}\left(\mathcal{N}_{3}\right)$ and $K_{5}$ are positive constants.

Finally, from (4.16), (4.17), (4.18), (4.19), (4.24) and (4.25), we infer

$$
\left\|e_{m+1}\right\|_{H}^{2}+\frac{k}{2} \sum_{n=0}^{m}\left\|e_{n}\right\|_{V}^{2}+\sum_{n=0}^{m}\left\|e_{n+1}-e_{n}\right\|_{H}^{2} \leq k K_{6}\left(\mathcal{N}_{3}\right) e^{K_{7}\left(\alpha_{1}+k_{0}\right)}
$$

where $K_{6}\left(\mathcal{N}_{3}\right)$ and $K_{7}$ are two positive constants.

(4) Estimate of $\left\|T(n k) u^{0}-T_{k}^{n} u^{0}\right\|_{V}$ for $\alpha_{0} / k \leq n \leq m+1$, when $T_{k}^{n} u^{0}$ and $T(n k) u^{0}$ belong to $\mathcal{N}_{3}$ for $0 \leq n \leq m$ and $0 \leq n k \leq m k+k_{0}$, respectively, where $\alpha_{0} / k<m \leq \alpha_{1} / k$. To this end, we at first estimate the term $\left\|t_{n}\left(T\left(t_{n}\right) u^{0}-T_{k}^{n} u^{0}\right)\right\|_{V}$ for $0 \leq n \leq m$. Formula $(4.1)_{k}$ gives

$$
\begin{gathered}
t_{n+1} u_{n+1}-t_{n} u_{n}+k A\left(\theta t_{n+1} u_{n+1}+(1-\theta) t_{n} u_{n}\right) \\
=k t_{n} f\left(u_{n}\right)+k u_{n+1}+\theta k^{2} A u_{n+1} .
\end{gathered}
$$

We set $\bar{e}_{n}=t_{n}\left(u_{n}-u\left(t_{n}\right)\right)$. From (4.27) and from the equation (4.1) we deduce

$$
\begin{aligned}
& \bar{e}_{n+1}-\bar{e}_{n}+k A\left(\theta \bar{e}_{n+1}+(1-\theta) \bar{e}_{n}\right) \\
& =k t_{n}\left(f\left(u_{n}\right)-f\left(u\left(t_{n}\right)\right)\right)-\int_{t_{n}}^{t_{n+1}}\left[\frac{d}{d s}(s u(s))-\left(\frac{d}{d s}(s u(s))\right)_{/ s=t_{n}}\right] d s \\
& \quad+\theta k A\left(t_{n} u\left(t_{n}\right)-t_{n+1} u\left(t_{n+1}\right)\right)+k e_{n}+k\left(u_{n+1}-u_{n}\right)+\theta k^{2} A u_{n+1} .
\end{aligned}
$$

Taking the inner product in $H$ of $(4.28)$ by $\bar{e}_{n+1}-\bar{e}_{n}$, we obtain

$$
\begin{aligned}
\| \bar{e}_{n+1}- & \bar{e}_{n}\left\|_{H}^{2}+\frac{k}{2}\right\| \bar{e}_{n+1}\left\|_{V}^{2}-\frac{k}{2}\right\| \bar{e}_{n}\left\|_{V}^{2}+\frac{k}{2}(2 \theta-1)\right\| \bar{e}_{n+1}-\bar{e}_{n} \|_{V}^{2} \\
\leq & k\left\|\bar{e}_{n+1}-\bar{e}_{n}\right\|_{H}\left[L\left\|\bar{e}_{n}\right\|_{V}+\left\|e_{n}\right\|_{H}+\left\|u_{n+1}-u_{n}\right\|_{H}\right] \\
& +k\left\|\bar{e}_{n+1}-\bar{e}_{n}\right\|_{V}\left[\theta k\left\|u_{n+1}\right\|_{V}+\theta\left\|t_{n} u\left(t_{n}\right)-t_{n+1} u\left(t_{n+1}\right)\right\|_{V}\right. \\
& \left.+\frac{1}{k}\left\|\int_{t_{n}}^{t_{n+1}}\left[\frac{d}{d s}(s u(s))-\left(\frac{d}{d s}(s u(s))\right)_{/ s=t_{n}}\right] d s\right\|_{V^{\prime}}\right]
\end{aligned}
$$


or also,

$$
\begin{aligned}
\left\|\bar{e}_{n+1}\right\|_{V}^{2}-\left\|\bar{e}_{n}\right\|_{V}^{2} \leq & 2 k L^{2}\left\|\bar{e}_{n}\right\|_{V}^{2}+2 k\left\|e_{n}\right\|_{H}^{2}+2 k\left\|u_{n+1}-u_{n}\right\|_{H}^{2} \\
& +\frac{3}{2 \theta-1}\left[k^{2}\left\|u_{n+1}\right\|_{V}^{2}+k \int_{t_{n}}^{t_{n+1}}\left\|\frac{d}{d s}(s u(s))\right\|_{V}^{2} d s\right. \\
& \left.+k \int_{t_{n}}^{t_{n+1}}\left\|\frac{d^{2}}{d s^{2}}(s u(s))\right\|_{V^{\prime}}^{2} d s\right] .
\end{aligned}
$$

Summing the previous inequality over $n$ and applying Lemma 4.2, we have

$$
\begin{aligned}
\max _{0 \leq n \leq m+1}\left\|\bar{e}_{n+1}\right\|_{V}^{2} & \\
\leq C \exp \left(2 k L^{2}(m+1)\right) & {\left[k \sum_{n=0}^{m}\left\|e_{n}\right\|_{H}^{2}+k \sum_{n=0}^{m}\left\|u_{n+1}-u_{n}\right\|_{H}^{2}\right.} \\
& +k^{2} \sum_{n=0}^{m}\left\|u_{n+1}\right\|_{V}^{2}+k \int_{0}^{t_{m+1}}\left\|\frac{d}{d s}(s u(s))\right\|_{V}^{2} d s \\
& \left.+k \int_{0}^{t_{m+1}}\left\|\frac{d^{2}}{d s^{2}}(s u(s))\right\|_{V^{\prime}}^{2} d s\right] .
\end{aligned}
$$

But

$$
k \sum_{n=0}^{m}\left\|u_{n+1}-u_{n}\right\|_{H}^{2} \leq 2 k \sum_{n=0}^{m}\left\|e_{n+1}-e_{n}\right\|_{H}^{2}+k^{2} \int_{0}^{t_{m+1}}\left\|\frac{d u}{d t}\right\|_{H}^{2} d s
$$

and

$$
k^{2} \sum_{n=0}^{m}\left\|u_{n+1}\right\|_{V}^{2} \leq k^{2}(m+1) B_{3}^{2} .
$$

Finally, we derive from (4.29), (4.30), (4.31), (4.26), as well as from (3.25), (4.20) and (4.25), that

$$
\max _{0 \leq n \leq m+1}\left\|\bar{e}_{n+1}\right\|_{V} \leq k^{1 / 2} K_{8}\left(\mathcal{N}_{3}\right) e^{K_{9}\left(\alpha_{1}+k_{0}\right)},
$$

where $K_{8}\left(\mathcal{N}_{3}\right)$ and $K_{9}$ are positive constants.

Hence, we have

$$
\max _{\alpha_{0} / k \leq n \leq m+1}\left\|T(n k) u^{0}-T_{k}^{n} u^{0}\right\|_{V} \leq \frac{k^{1 / 2}}{\alpha_{0}} K_{8}\left(\mathcal{N}_{3}\right) e^{K_{9}\left(\alpha_{1}+k_{0}\right)} .
$$

This completes the proof of Theorem 4.1.

Remark 4.2. If $f$ is locally Lipschitz continuous from $V$ into $H$ and from $H$ into $V^{\prime}$, we can improve the estimate (4.32) (and the proof is shorter). For estimates in the case where $f$ is globally Lipschitz continuous from $H$ into $H$, we refer the reader to Crouzeix and Thomée [8].

Remark 4.3. Now we consider a discretization in space and time of the equation (4.1). More precisely, if $\left(V_{h}\right)_{h}$ are the spaces given in Subsection 3.1, we define an approximation $u_{n}^{h} \in V_{h}$ of the solution $u$ of (4.1) at time $t_{n}$ by the recursion formula

$(4.1)_{k}^{h}\left\{\begin{array}{l}u_{n+1}^{h}=\left(1-(1-\theta) k A_{h}\right)\left(1+\theta k A_{h}\right)^{-1} u_{n}^{h}+k\left(1+\theta k A_{h}\right)^{-1} Q_{h} f\left(u_{n}^{h}\right), \\ u_{0 h}=u_{h}^{0} \in V_{h}\end{array}\right.$

(where $A_{h}$ and $Q_{h}$ are given in Subsection 3.1). 
Then in the same way as above, one proves that $(4.1)_{k}^{h}$ gives rise to a dynamical system $T_{k}^{h}$ which admits an attractor $A_{k}^{h}$. And $\delta_{V}\left(A_{k}^{h}, A\right) \rightarrow 0$ as $h$ and $k$ tend to 0 .

Furthermore, if we are in the situation described in Subsection 3.2 and if $k h^{-2 m} \leq$ $C$, where $C$ is a positive constant, one can define a dynamical system $\tilde{T}_{k}^{h}$ which admits an attractor $\tilde{A}_{k}^{h}$ in $Y^{\alpha}$ and $\delta_{Y^{\alpha}}\left(\tilde{A}_{k}^{h}, \mathcal{A}\right) \rightarrow 0$ as $h$ and $k$ tend to 0 .

5. A Remark on the Two-Dimensional Navier-Stokes Equations. Let $\Omega$ be a regular, bounded domain in $\mathbf{R}^{2}$. The Navier-Stokes equations for the velocity $u(x, t)=\left(u_{1}(x, t), u_{2}(x, t)\right)$ and the pressure $p(x, t)$ are

$$
\begin{cases}\frac{\partial u}{\partial t}-\nu \Delta u+\sum_{i=1}^{2} u_{i} \frac{\partial u}{\partial x_{i}}+\operatorname{grad} p=F & \text { in } \Omega \times \mathbf{R}_{+}, \\ \operatorname{div} u=0 & \text { in } \Omega \times \mathbf{R}_{+}, \\ u=0 & \text { on } \partial \Omega \times \mathbf{R}_{+}, \\ u(x, 0)=u_{0}(x) & \text { in } \Omega,\end{cases}
$$

where $F$ and $u_{0}$ are given and $\nu>0$ is the kinematic viscosity. Let us denote by $\mathbf{H}^{j}(\Omega)$ the space $\left(H^{j}(\Omega)\right)^{2}$ for $j=1$ or 2 and by $\mathrm{L}^{2}(\Omega)$ the space $\left(L^{2}(\Omega)\right)^{2}$. We consider the space

$$
\mathcal{V}=\left\{\phi \in\left(C_{0}^{\infty}(\Omega)\right)^{2} ; \operatorname{div} \phi=0\right\}
$$

and denote by $H$ and $V$ the closures of $\mathcal{V}$ in $\mathrm{L}^{2}(\Omega)$ and $\mathbf{H}^{1}(\Omega)$, respectively. The spaces $H$ and $V$ are provided with the inner products

$$
(u, v)=\sum_{j=1}^{2} \int_{\Omega} u_{j} v_{j} d x, \quad \text { and } \quad((u, v))=\sum_{j, k=1}^{2} \int_{\Omega} \frac{\partial u_{k}}{\partial x_{j}} \frac{\partial v_{k}}{\partial x_{j}} d x
$$

respectively, where $x=\left(x_{1}, x_{2}\right)$.

We also set $|u|=(u, u)^{1 / 2}$ and $\|u\|=((u, u))^{1 / 2}$ for $u$ in $H$ and $V$, respectively.

Let us denote by $P$ the orthogonal projection of $\mathrm{L}^{2}(\Omega)$ onto $H$. We define $A=-P \Delta$ to be the operator with domain $D(A)=\mathbf{H}^{2}(\Omega) \cap V$ acting in $H$ and use the same notation for its extension to an operator from $V$ into $V^{\prime}$. Since $A^{-1}$ is a compact selfadjoint linear operator in $H$, the spectrum of $A$ consists of an infinite sequence

$$
0<\lambda_{1} \leq \lambda_{2} \leq \cdots
$$

of eigenvalues (counted according to their multiplicities), $\lambda_{n} \rightarrow \infty$ as $n \rightarrow \infty$, and there exists an orthonormal basis $\left\{\phi_{n}\right\}_{n \geq 1}$ of $H$ such that

$$
A \phi_{n}=\lambda_{n} \phi_{n}, \quad n=1,2, \ldots
$$

For any $N \geq 1$ we denote by $P_{N}$ the orthogonal projection in $H$ (and in $V, V^{\prime}, D(A)$ ) onto the space $V_{N}$ spanned by $\phi_{1}, \phi_{2}, \ldots, \phi_{N}$. For $u=\left(u_{1}, u_{2}\right)$ and $v=\left(v_{1}, v_{2}\right)$ in $\mathbf{H}^{1}(\Omega)$ we define $B(u, v) \in V^{\prime}$ by

$$
(B(u, v), w)=\sum_{j, k=1}^{2} \int_{\Omega} u_{j} \frac{\partial v_{k}}{\partial x_{j}} w_{k} d x \quad \forall w \in V .
$$

Then $B$ is a bilinear continuous operator from $\mathbf{H}^{1}(\Omega) \times \mathbf{H}^{1}(\Omega)$ into $V^{\prime}$ and this operator can be extended as an operator from $\mathbf{H}^{m_{1}}(\Omega) \times \mathbf{H}^{m_{2}}(\Omega)$ into $V^{\prime}$ or $H$, for appropriate values of $m_{1}$ and $m_{2}$ (see Témam [32] for instance). 
Using the above notations, it can be shown that (5.1) is equivalent to the following initial value problem

$$
\left\{\begin{array}{l}
d u / d t+\nu A u+B(u, u)=f \quad \text { in } H \\
u(0)=u_{0}
\end{array}\right.
$$

where we assume that $f(x)=P F(x)$ and $u_{0}$ belong to $H$ and $V$, respectively (see [32] for further details). Let us point out that $f$ does not depend on $t$.

Now we introduce the map $T(t): V \rightarrow V, t \geq 0$, defined by $T(t) u_{0}=u(t)$, where $u(t)$ is the solution of (5.3). It is well known that $T(t) u_{0}$ exists for any $t \geq 0$ and any $u_{0} \in V$ and that $T(t)$ is a $C^{0}$-semigroup on $V$ (see Ladyzhenskaya [23], [24], for instance). In the same papers she also showed that $T(t) u_{0}$ has its $\varlimsup$ im as $t \rightarrow+\infty$ bounded by a constant independent of the initial data, i.e., $T(t)$ is point dissipative. Since $T(t)$ is compact for $t>0$, we deduce from a result of Billotti and LaSalle [2] that $T(t)$ admits a compact attractor $A$ which attracts bounded sets of $V$ (see also [12]).

Now let us consider the following differential system on the space $V_{N}$ spanned by $\phi_{1}, \phi_{2}, \ldots, \phi_{N}$ :

$$
\left\{\begin{array}{l}
d u_{N} / d t+\nu A u_{N}+P_{N} B\left(u_{N}, u_{N}\right)=P_{N} f(x), \\
u_{N}(0)=u_{0 N}
\end{array}\right.
$$

where $u_{0 N} \in V_{N}$. We introduce the map $T_{N}(t): V_{N} \rightarrow V_{N}, t \geq 0$, defined by $T_{N}(t) u_{0 N}=u_{N}(t)$, where $u_{N}(t)$ is the solution of $(5.3)_{N}$. As above, $T_{N}(t)$ is a $C^{0}$-semigroup on $V_{N}$ (see Témam [32] for instance). In [32, Section 14.2], it is also shown that $T_{N}(t) u_{0 N}$ has its $\varlimsup$ as $t \rightarrow+\infty$ bounded by a constant independent of the initial data and of $N$. Thus, by [2], $T_{N}(t)$ admits a compact attractor $A_{N}$ which attracts bounded sets of $V_{N}$. But thanks to Theorem 2.4 we obtain the following more precise result (for related results, see Constantin, Foias and Témam [6]).

THEOREM 5.1. For any $N \geq 1, T_{N}$ admits a compact attractor $A_{N}$ which attracts bounded sets of $V_{N}$. Moreover, $\delta_{V}\left(A_{N}, \mathcal{A}\right) \rightarrow 0$ as $N \rightarrow+\infty$.

Theorem 5.1 is a straightforward consequence of Lemma 2.1. In order to prove that $\left\|T(t) u_{N}-T_{N}(t) u_{N}\right\|_{V}$ satisfies the conditions (2.2) and (2.3), we use relations and inequalities which are similar to those contained in [32, Section 3.1]. In particular, we use the Young inequality, Gronwall's lemma technique (see [32, Formulas (3.10), (3.12)]), Lemmas 11.1 and 14.3 of [32] and the following inequality:

$$
\begin{array}{r}
|(B(u, v), w)| \leq C_{1}|u|^{1 / 2}\|u\|^{1 / 2}\|v\|^{1 / 2}|A v|^{1 / 2}|w| \\
\forall u \in V, v \in D(A), w \in H .
\end{array}
$$

The proof of Theorem 5.1 is given in Hale, Lin and Raugel [15].

Remark 5.1. The same kind of proof shows that if $A_{\nu_{0}}$ is the attractor associated with (5.3) for $\nu=\nu_{0}$, then $\delta_{V}\left(A_{\nu}, A_{\nu_{0}}\right) \rightarrow 0$ as $\nu \rightarrow \nu_{0}$, where $\nu_{0} \neq 0$.

Remark 5.2. The generalization of Theorem 5.1 to an approximation of the Navier-Stokes equations by a finite element method seems technically involved. In [19], Heywood and Rannacher have given uniform in time error estimates for the approximation of the Navier-Stokes equations by a finite element method when the solution is exponentially stable (see [18], [19] for the definition). The referee has called our attention to this recent paper ([19]) of Heywood and Rannacher and has 
pointed out that our method of proof of Lemma 2.1 has some analogy with their proof of Theorem 3.2 in [19].

6. Approximation of the Damped Wave Equation. Let $\Omega$ be a bounded domain in $\mathbf{R}^{3}$, $\alpha$ be a positive constant and consider the equation

$$
\begin{cases}\frac{\partial^{2} u}{\partial t^{2}}+2 \alpha \frac{\partial u}{\partial t}-\Delta u=-f(u)-g(x) & \text { in } \Omega \times(0, \infty) \\ u=0 & \text { on } \partial \Omega \\ \left.\left(u, \frac{\partial u}{\partial t}\right)\right|_{t=0}=(\phi, \psi), & \end{cases}
$$

where $g$ belongs to $L^{2}(\Omega)$ and $(\phi, \psi)$ belongs to the space $X \equiv H_{0}^{1}(\Omega) \times L^{2}(\Omega)$. We assume that the boundary $\partial \Omega$ of $\Omega$ is smooth enough or that $\Omega$ is a convex domain. Furthermore, we suppose that $f \in C^{3}(\mathbf{R})$ and that there are constants $\gamma \geq 0, C_{1}>0$, with $0 \leq \gamma<2$, such that

$$
\left\{\begin{array}{l}
|f(v)| \leq C_{1}\left(|v|^{1+\gamma}+1\right), \quad\left|f^{\prime}(v)\right| \leq C_{1}\left(|v|^{\gamma}+1\right), \\
\left|f^{\prime \prime}(v)\right| \leq C_{1}(|v|+1),\left|f^{\prime \prime \prime}(v)\right| \leq C_{1} \quad \text { for all } v \in \mathbf{R} .
\end{array}\right.
$$

Inequalities (6.2) imply that the map $f: \phi \in H_{0}^{1}(\Omega) \rightarrow f(\phi(x)) \in L^{2}(\Omega)$ is a compact $C^{2}$-mapping from $H_{0}^{1}(\Omega)$ into $L^{2}(\Omega)$. Henceforth, we equip the space $X$ with the norm

$$
\|(\phi, \psi)\|_{X}=\left(\|\phi\|_{H_{0}^{1}(\Omega)}^{2}+\|\psi\|_{L^{2}(\Omega)}^{2}\right)^{1 / 2} \quad \forall(\phi, \psi) \in X .
$$

As it was proved in Babin and Vishik [1], for any $(\phi, \psi) \in X$, Problem (6.1) has a unique solution $u(t)$, for $t \geq 0$, and the pair $(u, \partial u / \partial t)$ belongs to $C^{0}([0,+\infty) ; X)$. Furthermore, if we set $T(t)(\phi, \psi)=(u(t), \partial u(t) / \partial t)$, for $t \geq 0$, then $T(t): X \rightarrow$ $X, t \geq 0$, is a $C^{0}$-semigroup on $X$.

Now suppose there is a constant $C>0$ so that $f$ satisfies

$$
f(v) v>-C, f^{\prime}(v)>-C \quad \text { for all } v \in \mathbf{R} .
$$

Let us introduce the Liapunov functional $V$ given by

$$
V(\phi, \psi)=\int_{\Omega}\left[\frac{1}{2}|\nabla \phi(x)|^{2}+\frac{1}{2} \psi^{2}(x)+F(\phi(x))+g(x) \phi(x)\right] d x,
$$

for all $(\phi, \psi) \in X$, where $F(v)=\int_{0}^{v} f(s) d s$. It was proved in Babin and Vishik [1] that

$$
\left\{\begin{array}{l}
V(\phi, \psi) \geq \frac{1}{2}\|\psi\|_{L^{2}(\Omega)}^{2}+\|\phi\|_{H_{0}^{1}(\Omega)}^{2}-C_{2} \\
V(\phi, \psi) \leq \frac{1}{2}\|\psi\|_{L^{2}(\Omega)}^{2}+C_{3}\|\phi\|_{H_{0}^{1}(\Omega)}^{4}+C_{4}
\end{array}\right.
$$

where $C_{2}, C_{3}, C_{4}$ are some fixed positive constants, and that, for $t \geq \tau$ and for any solution $u$ of $(6.1)$,

$$
V\left(u(t, \cdot), \frac{\partial u}{\partial t}(t, \cdot)\right)-V\left(u(\tau, \cdot), \frac{\partial u}{\partial t}(\tau, \cdot)\right)=-2 \alpha \int_{\tau}^{t} \int_{\Omega}\left[\frac{\partial u}{\partial t}(s, x)\right]^{2} d x d s
$$

The properties (6.5), (6.6) imply that the orbits of bounded sets are bounded. In particular, there exist two functions $C_{0}(R)$ and $C_{1}(R)$ of $R$ such that, if

$$
\|(\phi, \psi)\|_{X} \leq R
$$


then

$$
V(T(t)(\phi, \psi)) \leq V(\phi, \psi) \leq C_{0}(R) \quad \forall t \in R
$$

and

$$
\|T(t)(\phi, \psi)\|_{X} \leq C_{1}(R) \quad \forall t \in R .
$$

Moreover, it was shown in Hale [12] that $T(t)$ is point dissipative and is an $\alpha$ contraction. Therefore, in view of a result of Massatt [27], $T(t)$ admits a compact attractor $A$ in $X$ which attracts bounded sets of $X$ (see Hale [12, Theorem 6.1]).

6.1. Approximation By a Special Projection Method. Let us recall that the spectrum of the operator $-\Delta$ with domain $D(-\Delta)=H^{2}(\Omega) \cap H_{0}^{1}(\Omega)$ consists of an infinite sequence

$$
0<\lambda_{1} \leq \lambda_{2} \leq \cdots
$$

of eigenvalues (counted according to their multiplicities), $\lambda_{n} \rightarrow+\infty$ as $n \rightarrow+\infty$, and that there exists an orthornormal basis $\left\{w_{n}\right\}_{n \geq 1}$ of $L^{2}(\Omega)$ such that

$$
-\Delta w_{n}=\lambda_{n} w_{n}
$$

Note that $\left\{\lambda_{n}^{-1 / 2} w_{n}\right\}_{n \geq 1}$ is an orthonormal basis of $H_{0}^{1}(\Omega)$. For any $N \geq 1$ we denote by $P_{N}$ the orthogonal projection in $L^{2}(\Omega)$ (and in $H_{0}^{1}(\Omega)$ ) onto the space $V_{N}$ spanned by $w_{1}, w_{2}, \ldots, w_{N}$, and we consider the following equation in $V_{N}$ :

$$
\left\{\begin{array}{l}
\frac{\partial^{2} u_{N}}{\partial t^{2}}+2 \alpha \frac{\partial u_{N}}{\partial t}-\Delta u_{N}=-P_{N} f\left(u_{N}\right)-P_{N} g(x), \\
\left.\left(u_{N}, \frac{\partial u_{N}}{\partial t}\right)\right|_{t=0}=\left(\phi_{N}, \psi_{N}\right),
\end{array}\right.
$$

where $\left(\phi_{N}, \psi_{N}\right)$ belongs to the space $X_{N}=V_{N} \times V_{N}$. We can prove, as for Problem (6.1), that, for any $\left(\phi_{N}, \psi_{N}\right)$ in $X_{N}$, the equation $(6.1)_{N}$ has a unique solution $u_{N}(t)$ for $t \geq 0$. Moreover, if we set $T_{N}(t)\left(\phi_{N}, \psi_{N}\right)=\left(u_{N}(t), \partial u_{N}(t) / \partial t\right)$ for $t \geq 0$, then $T_{N}(t): X_{N} \rightarrow X_{N}, t \geq 0$, is a $C^{0}$-semigroup on $X_{N}$.

THEOREM 6.1. For any $N \geq 1, T_{N}$ admits a compact attractor $A_{N}$ which attracts bounded sets of $X_{N}$. Moreover, $\delta_{X}\left(A_{N}, A\right) \rightarrow 0$ as $N \rightarrow+\infty$.

Proof. (1) We at once verify that, for $t \geq \tau$, for any solution $u_{N}$ of Eq. $(6.1)_{N}$,

$$
\begin{gathered}
V\left(u_{N}(t, \cdot), \frac{\partial u_{N}}{\partial t}(t, \cdot)\right)-V\left(u_{N}(\tau, \cdot), \frac{\partial u_{N}}{\partial t}(\tau, \cdot)\right) \\
=-2 \alpha \int_{\tau}^{t} \int_{\Omega}\left(\frac{\partial u_{N}}{\partial t}(s, x)\right)^{2} d x d s .
\end{gathered}
$$

The estimates (6.5) imply that the orbits of bounded sets are bounded independently of $N$. In particular, $T_{N}(t)\left(\phi_{N}, \psi_{N}\right)$ satisfies the estimates (6.8) and (6.9), for any $\left(\phi_{N}, \psi_{N}\right)$ satisfying (6.7). As $T_{N}(t)$ is compact, the orbit through $\left(\phi_{N}, \psi_{N}\right)$ is precompact and its $\omega$-limit set must be an invariant set. Relation (6.11) implies that its $\omega$-limit set belongs to the set $E_{N}$ of the equilibrium points. Using the condition (6.4), one easily proves that there exists a constant $r_{0}>0$ such that

$$
\forall N \geq 1, \quad E_{N} \subset B_{X}\left(r_{0}\right),
$$

where, for any $r>0, B_{X}(r)=\left\{(\phi, \psi) \in X:\|(\phi, \psi)\|_{X}<r\right\}$. Let us also set $B_{X_{N}}(r)=B_{X}(r) \cap X_{N}$. Then, for $r_{1}=2 r_{0}$, the ball $B_{X}\left(r_{1}\right)$ attracts all points 
of $X_{N}$ (i.e., for any $\left(\phi_{N}, \psi_{N}\right) \in X_{N}$, there exists $t_{N} \geq 0$ such that, for $t \geq t_{N}$, $\left.T_{N}(t)\left(\phi_{N}, \psi_{N}\right) \in B_{X_{N}}\left(r_{1}\right)\right)$. We remark that the orbit of $B_{X_{N}}\left(r_{1}\right)$ is included in $B_{X_{N}}\left(C_{1}\left(r_{1}\right)\right)$, where $C_{1}\left(r_{1}\right)$ is given by (6.9), and that $B_{X_{N}}\left(C_{1}\left(r_{1}\right)\right)$ attracts a neighborhood of any point and, hence, all compact sets of $X_{N}$. We now set $R_{0}=C_{1}\left(C_{1}\left(r_{1}\right)\right)$. Arguing as in Hale [11, Theorem 2.1], one finally shows that $T_{N}(t)$ admits a compact attractor $A_{N}$ which attracts bounded sets of $X_{N}$ and is included in the ball $B_{X}\left(R_{0}\right) \cap X_{N}$.

(2) In order to prove that $\delta_{X}\left(A_{N}, A\right) \rightarrow 0$ as $N \rightarrow+\infty$, we show that the hypotheses of Lemma 2.1 hold. Let $\mathcal{N}_{1} \equiv B_{X}\left(R_{1}\right)$ be a neighborhood of $A$. We shall prove that $T_{N}(t)$ approximates $T(t)$ on $\mathcal{N}_{1}$ uniformly on compact sets of $[0,+\infty)$. Let $t_{1}$ be any positive real number. We first estimate

$$
\left\|\left(u(t)-P_{N} u(t), \frac{\partial u}{\partial t}(t)-\frac{\partial P_{N} u}{\partial t}(t)\right)\right\|_{X}
$$

for $0 \leq t \leq t_{1}$, where $(u(t), \partial u(t) / \partial t)=T(t)\left(\phi_{N}, \psi_{N}\right)$ and $\left(\phi_{N}, \psi_{N}\right) \in \mathcal{N}_{1} \cap X_{N}$. We have

$$
\begin{gathered}
\frac{\partial^{2}}{\partial t^{2}}\left(u-P_{N} u\right)+2 \alpha \frac{\partial}{\partial t}\left(u-P_{N} u\right)-\Delta\left(u-P_{N} u\right) \\
=-\left(I-P_{N}\right) f(u)-\left(I-P_{N}\right) g(x)
\end{gathered}
$$

Taking the inner product in $L^{2}(\Omega)$ of $(6.13)$ by $\partial\left(u-P_{N} u\right) / \partial t$, we get after an integration from 0 to $t$,

$$
\begin{aligned}
& \left\|\frac{\partial}{\partial t}\left(u-P_{N} u\right)(t)\right\|_{L^{2}(\Omega)}^{2}+\left\|u(t)-P_{N} u(t)\right\|_{H_{0}^{1}(\Omega)}^{2} \\
& \quad \leq \frac{t_{1}}{\alpha}\left(\sup _{s \in\left[0, t_{1}\right]}\left\|\left(I-P_{N}\right) f(u(s))\right\|_{L^{2}(\Omega)}^{2}+\left\|\left(I-P_{N}\right) g(x)\right\|_{L^{2}(\Omega)}^{2}\right) .
\end{aligned}
$$

Since $f$ is a compact mapping from $H_{0}^{1}(\Omega)$ into $L^{2}(\Omega)$ and $u(s), 0 \leq s \leq t$, belongs to the bounded set $\bar{B}\left(C_{1}\left(R_{1}\right)\right)=\left\{v \in H_{0}^{1}(\Omega):\|v\|_{H_{0}^{1}(\Omega)} \leq C_{1}\left(R_{1}\right)\right\}$, we deduce from (6.14) that, for $0 \leq t \leq t_{1}$,

$$
\left\|\frac{\partial}{\partial t}\left(u-P_{N} u\right)(t)\right\|_{L^{2}(\Omega)}^{2}+\left\|u(t)-P_{N} u(t)\right\|_{H_{0}^{1}(\Omega)}^{2} \leq \eta_{1}\left(N, t_{1}, C_{1}\left(R_{1}\right)\right),
$$

where

$$
\lim _{N \rightarrow+\infty} \eta_{1}\left(N, t_{1}, C_{1}\left(R_{1}\right)\right)=0
$$

Now we estimate

$$
\left\|\left(P_{N} u(t)-u_{N}(t), \frac{\partial}{\partial t}\left(P_{N} u(t)-u_{N}(t)\right)\right)\right\|_{X}
$$

for $0 \leq t \leq t_{1}$, where $\left(u_{N}(t), \partial u_{N}(t) / \partial t\right)=T_{N}(t)\left(\phi_{N}, \psi_{N}\right)$. The function $u_{N}-P_{N} u$ satisfies the equation

$$
\frac{\partial^{2}}{\partial t^{2}}\left(u_{N}-P_{N} u\right)+2 \alpha \frac{\partial}{\partial t}\left(u_{N}-P_{N} u\right)-\Delta\left(u_{N}-P_{N} u\right)=P_{N}\left(f(u)-f\left(u_{N}\right)\right)
$$


Taking the inner product in $L^{2}(\Omega)$ of $(6.17)$ with $\partial\left(u_{N}-P_{N} u\right) / \partial t$, we obtain

$$
\begin{gathered}
\frac{1}{2} \frac{\partial}{\partial t}\left(\left\|\frac{\partial}{\partial t}\left(u_{N}-P_{N} u\right)\right\|_{L^{2}(\Omega)}^{2}\right)+\frac{1}{2} \frac{\partial}{\partial t}\left(\left\|u_{N}-P_{N} u\right\|_{H_{0}^{1}(\Omega)}^{2}\right) \\
\leq \frac{L^{2}}{2 \alpha}\left\|u_{N}-P_{N} u\right\|_{H_{0}^{1}(\Omega)}^{2}+\frac{L^{2}}{2 \alpha}\left\|u-P_{N} u\right\|_{H_{0}^{1}(\Omega)}^{2},
\end{gathered}
$$

where $L>0$ is the Lipschitz constant of $f$ in the ball $\bar{B}\left(C_{1}\left(R_{1}\right)\right)$. Now using Gronwall's lemma, we derive from (6.18) as well as from (6.15) that, for $0 \leq s \leq t$,

$$
\begin{aligned}
& \left\|\frac{\partial}{\partial t}\left(u_{N}-P_{N} u\right)(t)\right\|_{L^{2}(\Omega)}^{2}+\left\|u_{N}(t)-P_{N} u(t)\right\|_{H_{0}^{1}(\Omega)}^{2} \\
& \leq e^{t_{1} L^{2} / 2 \alpha} \frac{L^{2}}{2 \alpha} \eta_{1}\left(N, t_{1}, C_{1}\left(R_{1}\right)\right) .
\end{aligned}
$$

The estimates (6.15), (6.16) and (6.19) show that $T_{N}(t)$ approximates $T(t)$ on $\mathcal{N}_{1}$ uniformly on compact sets of $[0,+\infty)$.

6.2. A More General Galerkin Method. Let $h>0$ be a real parameter which will tend to 0 and $\left(V_{h}\right)_{h}$ be a family of finite-dimensional subspaces of $H_{0}^{1}(\Omega)$. We denote by $[\cdot, \cdot]$ the inner product of $L^{2}(\Omega)$ and by $a(\cdot, \cdot)$ the inner product of $H_{0}^{1}(\Omega)$, i.e.,

$$
\forall v \in H_{0}^{1}(\Omega), \forall w \in H_{0}^{1}(\Omega), \quad a(v, w)=\int_{\Omega} \nabla v \nabla w d x
$$

As in Subsection 3.1, we denote by $Q_{h} \in \mathcal{L}\left(L^{2}(\Omega) ; V_{h}\right)$ and $P_{h} \in \mathcal{L}\left(H_{0}^{1}(\Omega) ; V_{h}\right)$ the orthogonal projectors on $V_{h}$ in the spaces $L^{2}(\Omega)$ and $H_{0}^{1}(\Omega)$, respectively. We also introduce the operator $A_{h} \in \mathcal{L}\left(V_{h} ; V_{h}\right)$ defined by

$$
\forall v_{h} \in V_{h}, \quad\left(A_{h} w_{h}, v_{h}\right)=a\left(w_{h}, v_{h}\right) \quad \text { for } w_{h} \in V_{h} .
$$

We consider the following equation in $V_{h}$ :

$$
\left\{\begin{array}{l}
\frac{\partial^{2} u_{h}}{\partial t^{2}}+2 \alpha \frac{\partial u_{h}}{\partial t}+A_{h} u_{h}=-Q_{h} f\left(u_{h}\right)-Q_{h} g(x), \\
\left.\left(u_{h}, \frac{\partial u_{h}}{\partial t}\right)\right|_{t=0}=\left(\phi_{h}, \psi_{h}\right),
\end{array}\right.
$$

where $\left(\phi_{h}, \psi_{h}\right)$ belongs to the space $X_{h}=V_{h} \times V_{h}$. As in Subsection 6.1, we introduce the map $T_{h}(t): X_{h} \rightarrow X_{h}$, for $t \geq 0$, defined by

$$
T_{h}\left(\phi_{h}, \psi_{h}\right)=\left(u_{h}(t), \partial u_{h}(t) / \partial t\right)
$$

where $u_{h}$ is the solution of $(6.1)_{h}$. So we obtain a $C^{0}$-semigroup on $X_{h}$. As in Subsection 3.1, we need some additional hypotheses on the spaces $\left(V_{h}\right)_{h}$ :

there exists a constant $K_{0}>0$, independent of $h$, such that, for any $h>0$,

$$
\left\|Q_{h}\right\|_{\mathcal{L}\left(H_{0}^{1}(\Omega) ; H_{0}^{1}(\Omega)\right.} \leq K_{0}
$$

and

there exist two constants $K_{1}>0$ and $\theta>0$, independent of $h$, such that, for any $w$ in $H_{0}^{1}(\Omega)$,

$$
\left\|w-P_{h} w\right\|_{L^{2}(\Omega)}+\left\|w-Q_{h} w\right\|_{L^{2}(\Omega)} \leq K_{1} h^{\theta}\|w\|_{H_{0}^{1}(\Omega)} .
$$


(Usually, $\theta$ is taken equal to 1.) Finally, we introduce the Hilbert space $Y \equiv$ $L^{2}(\Omega) \times H^{-1}(\Omega)$, normed by $\|(\phi, \psi)\|_{Y}=\left(\|\phi\|_{L^{2}}^{2}+\|\psi\|_{H^{-1}(\Omega)}^{2}\right)^{1 / 2}$. Below we denote by $[\cdot, \cdot]$ the inner product in $L^{2}(\Omega)$. Now we are able to prove the following result.

THEOREM 6.2. For any $h>0, T_{h}$ admits a compact attractor $A_{h}$ which attracts bounded sets of $X_{h}$ and is contained in the ball $B_{X}\left(R_{0}\right) \cap X_{h}$, where $R_{0}$ is a constant independent of $h$. Moreover, $\delta_{Y}\left(A_{h}, A\right) \rightarrow 0$ as $h \rightarrow 0$.

Remark 6.1. In Section 3 we proved that $\delta_{V}\left(A_{h}, A\right) \rightarrow 0$ as $h \rightarrow 0$. Here, we can no longer prove that $\delta_{X}\left(A_{h}, A\right) \rightarrow 0$ as $h \rightarrow 0$, because $T(t)$ has no longer a smoothing action.

Proof of Theorem 6.2. (1) First we show in the same way as in the proof of Theorem 6.1 that, for any $h>0, T_{h}$ admits a compact attractor $A_{h}$ which attracts bounded sets of $X_{h}$ and is contained in $B_{X}\left(R_{0}\right) \cap X_{h}$, where $R_{0}$ is a constant independent of $h$. Note that $R_{0}$ can be chosen so that $A$ is also contained in $B_{X}\left(R_{0}\right)$.

(2) Now we check that, for any $r>0$, there exists a constant $L(r)>0$ such that, for all $v$ and $w$ in the ball $\bar{B}(r)=\left\{v \in H_{0}^{1}(\Omega):\|v\|_{H_{0}^{1}(\Omega)} \leq r\right\}$, we have

$$
\|f(v)-f(w)\|_{H^{-1}(\Omega)} \leq L(r)\|v-w\|_{L^{2}(\Omega)} .
$$

Indeed, we can write

$$
\begin{aligned}
\| f(v) & -f(w) \|_{H^{-1}(\Omega)}=\sup _{\Phi \in H_{0}^{1}(\Omega)} \frac{\int_{\Omega}(f(v(x))-f(w(x))) \Phi(x) d x}{\|\Phi\|_{H_{0}^{1}(\Omega)}} \\
& \leq \sup _{\Phi \in H_{0}^{1}(\Omega)} \frac{\int_{\Omega} \int_{0}^{1} f^{\prime}(w(x)+\tau(v(x)-w(x)))(v(x)-w(x)) \Phi(x) d x d \tau}{\|\Phi\|_{H_{0}^{1}(\Omega)}} .
\end{aligned}
$$

Hence, using the hypothesis $(6.2)$, we obtain

$$
\begin{aligned}
\|f(v)-f(w)\|_{H^{-1}(\Omega)} & \\
\leq \sup _{\Phi \in H_{0}^{1}(\Omega)} \frac{C_{1}}{\|\Phi\|_{H_{0}^{1}(\Omega)}}\{ & \left(\int_{\Omega}|v(x)-w(x)|^{2} d x\right)^{1 / 2} \\
& \times\left(\int_{\Omega} 2\left(|v(x)|^{\beta}+|w(x)|^{\beta}+1\right) d x\right) \\
& \left.\times\left(\int_{\Omega}|\Phi(x)|^{6} d x\right)^{1 / 6}\right\},
\end{aligned}
$$

where $\beta=\sup (3,6-3 \gamma)$. As $H_{0}^{1}(\Omega) \hookrightarrow L^{6}(\Omega)$, the property $(6.22)$ is a direct consequence of $(6.23)$.

(3) Now, for any $t_{1}>0$, we estimate $\left\|\left(u(t)-u_{h}(t), \partial u(t) / \partial t-\partial u_{h}(t) / \partial t\right)\right\|_{Y}$ for $0 \leq t \leq t_{1}$, where $u(t)$ and $u_{h}(t)$ are the solutions of the equations (6.1) and $(6.1)_{h}$, respectively, with initial condition $\left(\phi_{h}, \psi_{h}\right) \in B_{X}\left(R_{0}\right)$. Thanks to the hypothesis (6.21)(ii), we have, on the one hand,

$$
\left\|u(t)-Q_{h} u(t)\right\|_{L^{2}(\Omega)} \leq K_{1} h^{\theta} C_{1}\left(R_{0}\right),
$$


and, on the other hand,

$$
\begin{aligned}
\left\|\frac{\partial u}{\partial t}(t)-\frac{\partial}{\partial t} Q_{h} u(t)\right\|_{H^{-1}(\Omega)} & =\sup _{v \in H_{0}^{1}(\Omega)}\left[\frac{\partial u}{\partial t}-\frac{\partial}{\partial t} Q_{h} u, v-Q_{h} v\right] /\|v\|_{H_{0}^{1}(\Omega)} \\
& \leq \sup _{v \in H_{0}^{1}(\Omega)}\left\|\frac{\partial u}{\partial t}-\frac{\partial}{\partial t} Q_{h} u\right\|_{L^{2}(\Omega)} \frac{\left\|v-Q_{h} v\right\|_{L^{2}(\Omega)}}{\|v\|_{H_{0}^{1}(\Omega)}}
\end{aligned}
$$

which gives

$$
\left\|\frac{\partial u}{\partial t}(t)-\frac{\partial}{\partial t} Q_{h} u(t)\right\|_{H^{-1}(\Omega)} \leq 2 K_{1} h^{\theta} C_{1}\left(R_{0}\right)
$$

It remains to estimate the term $\left\|\left(Q_{h} u(t)-u_{h}(t), \partial Q_{h} u(t) / \partial t-\partial u_{h}(t) / \partial t\right)\right\|_{Y}$ for $0 \leq t \leq t_{1}$. Note that by $(6.21)(\mathrm{i}), Q_{h}$ can be extended to a continuous, linear operator from $H^{-1}(\Omega)$ into $V_{h}$ and that the element $u_{h}-Q_{h} u$ thus satisfies the equation

$$
\begin{gathered}
\frac{\partial^{2}}{\partial t^{2}}\left(u_{h}-Q_{h} u\right)+2 \alpha \frac{\partial}{\partial t}\left(u_{h}-Q_{h} u\right)+A_{h}\left(u_{h}-Q_{h} u\right) \\
=-Q_{h}\left(f\left(u_{h}\right)-f(u)\right)-\left(A_{h} Q_{h}-Q_{h} A\right) u .
\end{gathered}
$$

We now introduce the operator $S_{h} \in \mathcal{L}\left(H^{-1}(\Omega) ; V_{h}\right)$ given by

$$
\forall f \in H^{-1}(\Omega), \quad a\left(S_{h} f, v_{h}\right)=\left[f, v_{h}\right], \quad \forall v_{h} \in V_{h} .
$$

Clearly, one has

$$
\left\|S_{h} f\right\|_{H_{0}^{1}(\Omega)} \leq c\|f\|_{H^{-1}(\Omega)}
$$

where $c>0$ is a constant independent of $h$.

Taking the inner product in $L^{2}(\Omega)$ of $(6.26)$ by $S_{h}\left(\partial\left(u_{h}-Q_{h} u\right) / \partial t\right)$ and using the relation (6.27), we obtain

$$
\begin{aligned}
a\left(\frac{\partial^{2}}{\partial t^{2}} S_{h}\left(u_{h}-Q_{h} u\right), \frac{\partial}{\partial t} S_{h}\left(u_{h}-Q_{h} u\right)\right) \\
+2 \alpha a\left(S_{h} \frac{\partial}{\partial t}\left(u_{h}-Q_{h} u\right), S_{h} \frac{\partial}{\partial t}\left(u_{h}-Q_{h} u\right)\right) \\
+a\left(u_{h}-Q_{h} u, S_{h} \frac{\partial}{\partial t}\left(u_{h}-Q_{h} u\right)\right) \\
=-\left[f\left(u_{h}\right)-f(u), S_{h} \frac{\partial}{\partial t}\left(u_{h}-Q_{h} u\right)\right] \\
+a\left(u-Q_{h} u, S_{h} \frac{\partial}{\partial t}\left(u_{h}-Q_{h} u\right)\right) .
\end{aligned}
$$

But

$$
\begin{gathered}
a\left(u-Q_{h} u, S_{h} \frac{\partial}{\partial t}\left(u_{h}-Q_{h} u\right)\right)=a\left(P_{h} u-Q_{h} u, S_{h} \frac{\partial}{\partial t}\left(u_{h}-Q_{h} u\right)\right) \\
=\left[P_{h} u-Q_{h} u, \frac{\partial}{\partial t}\left(u_{h}-Q_{h} u\right)\right]=\left[P_{h} u-u, \frac{\partial}{\partial t}\left(u_{h}-Q_{h} u\right)\right]
\end{gathered}
$$

and

$$
a\left(u_{h}-Q_{h} u, S_{h} \frac{\partial}{\partial t}\left(u_{h}-Q_{h} u\right)\right)=\left[u_{h}-Q_{h} u, \frac{\partial}{\partial t}\left(u_{h}-Q_{h} u\right)\right]
$$


Then, from (6.29) we can derive the following inequality:

$$
\begin{aligned}
& \frac{1}{2} \frac{\partial}{\partial t}\left\|\frac{\partial}{\partial t} S_{h}\left(u_{h}-Q_{h} u\right)\right\|_{H_{0}^{1}(\Omega)}^{2}+2 \alpha\left\|\frac{\partial}{\partial t} S_{h}\left(u_{h}-Q_{h} u\right)\right\|_{H_{0}^{1}(\Omega)}^{2} \\
&+\frac{1}{2} \frac{\partial}{\partial t}\left\|u_{h}-Q_{h} u\right\|_{L^{2}(\Omega)}^{2} \\
& \leq\left\|f(u)-f\left(u_{h}\right)\right\|_{H^{-1}(\Omega)}\left\|\frac{\partial}{\partial t} S_{h}\left(u_{h}-Q_{h} u\right)\right\|_{H_{0}^{1}(\Omega)} \\
&+\left\|u-P_{h} u\right\|_{L^{2}(\Omega)}\left\|\frac{\partial}{\partial t}\left(u_{h}-Q_{h} u\right)\right\|_{L^{2}(\Omega)}
\end{aligned}
$$

Using the property $(6.22)$ and the fact that $(u, \partial u / \partial t)$ and $\left(u_{h}, \partial u_{h} / \partial t\right)$ belong to $B_{X}\left(C_{1}\left(R_{0}\right)\right)$, we infer from the above estimate that

$$
\begin{aligned}
\frac{\partial}{\partial t} \| & \frac{\partial}{\partial t} S_{h}\left(u_{h}-Q_{h} u\right)\left\|_{H_{0}^{1}(\Omega)}^{2}+\frac{\partial}{\partial t}\right\| u_{h}-Q_{h} u \|_{L^{2}(\Omega)}^{2} \\
\leq & \frac{L^{2}\left(C_{1}\left(R_{0}\right)\right)}{\alpha}\left\{\left\|u-Q_{h} u\right\|_{L^{2}(\Omega)}^{2}+\left\|u_{h}-Q_{h} u\right\|_{L^{2}(\Omega)}^{2}\right\} \\
& +2 C_{1}\left(R_{0}\right)\left\|u-P_{h} u\right\|_{L^{2}(\Omega)} .
\end{aligned}
$$

Integrating (6.30) from 0 to $t$ and using Gronwall's lemma as well as the hypothesis (6.21)(ii), we get, for $0 \leq t \leq t_{1}$,

$$
\left\|\frac{\partial}{\partial t} S_{h}\left(u_{h}-Q_{h} u\right)(t)\right\|_{H_{0}^{1}(\Omega)}^{2}+\left\|\left(u_{h}-Q_{h} u\right)(t)\right\|_{L^{2}(\Omega)}^{2} \leq K_{2} t_{1} e^{K_{3} t_{1}} h^{\theta}
$$

where $K_{2}>0$ and $K_{3}>0$ are two constants depending on $R_{0}$ only. Now we remark that

$$
\begin{aligned}
\left\|\frac{\partial}{\partial t}\left(u_{h}-Q_{h} u\right)\right\|_{H^{-1}(\Omega)} & =\sup _{v \in H_{0}^{1}(\Omega)}\left[\frac{\partial}{\partial t}\left(u_{h}-Q_{h} u\right), v\right] /\|v\|_{H_{0}^{1}(\Omega)} \\
& =\sup _{v \in H_{0}^{1}(\Omega)}\left[\frac{\partial}{\partial t}\left(u_{h}-Q_{h} u\right), Q_{h} v\right] /\|v\|_{H_{0}^{1}(\Omega)} \\
& =\sup _{v \in H_{0}^{1}(\Omega)} a\left(\frac{\partial}{\partial t} S_{h}\left(u_{h}-Q_{h} u\right), Q_{h} v\right) /\|v\|_{H_{0}^{1}(\Omega)}
\end{aligned}
$$

and therefore, thanks to the hypothesis $(6.21)(\mathrm{i})$,

$$
\left\|\frac{\partial}{\partial t}\left(u_{h}-Q_{h} u\right)\right\|_{H^{-1}(\Omega)} \leq K_{0}\left\|\frac{\partial}{\partial t} S_{h}\left(u_{h}-Q_{h} u\right)\right\|_{H_{0}^{1}(\Omega)} .
$$

Finally, by (6.24), (6.25), (6.31) and (6.32), we obtain, for $0 \leq t \leq t_{1}$,

$$
\left\|\left(u(t)-u_{h}(t), \frac{\partial u}{\partial t}(t)-\frac{\partial u_{h}}{\partial t}(t)\right)\right\|_{Y} \leq K_{4} t_{1}^{1 / 2} e^{K_{5} t_{1}} h^{\theta / 2}
$$

where $K_{4}$ and $K_{5}$ are positive constants depending on $R_{0}$ only.

(4) Since, for any $h>0, A_{h} \subset B_{X}\left(R_{0}\right)$, we deduce from the property (6.33), by arguing as in the proof of Proposition 2.10 (or in Remark 2.7), that, for any $\varepsilon_{0}>0$, there exists $h_{0}>0$ such that, for $h \leq h_{0}, \delta_{Y}\left(A_{h}, A\right) \leq \varepsilon_{0}$. 
Remark 6.2. The results of Theorems 6.1 and 6.2 extend easily to the cases where $\Omega$ is a bounded domain in $\mathbf{R}$ or $\mathbf{R}^{2}$ (for the conditions on $f$, see Babin and Vishik [1] or Hale [12]).

Lefschetz Center for Dynamical Systems

Division of Applied Mathematics

Brown University

Providence, Rhode Island 02912

Department of Mathematics

Michigan State University

East Lansing, Michigan 48823

Ecole Polytechnique

Centre de Mathématiques Appliquées

Unité de Recherche Associée au CNRS-756

91128 Palaiseau Cedex, France

1. A. V. BABIN \& M. I. VISHIK, "Regular attractors of semigroups and evolution equations," J. Math. Pures Appl., v. 62, 1983, pp. 442-491.

2. J. E. Billot v. 77, 1971, pp. 1082-1088.

3. J. H. Bramble, A. H. Schatz, V. ThoméE \& L. B. Wahlbin, "Some convergence estimates for semidiscrete Galerkin type approximations for parabolic equations," SIAM J. Numer. Anal., v. 14, 1977, pp. 218-241.

4. P. Brenner, M. Crouzeix \& V. Thomée, "Single step methods for inhomogeneous linear differential equations in Banach spaces," RAIRO Anal. Numér., v. 16, 1982, pp. 5-26.

5. P. G. Ciarlet The Finite Element Method for Elliptic Problems, North-Holland, Amsterdam, 1978.

6. P. Constantin, C. Foias \& R. TÉmam, "On the large time Galerkin approximation of the Navier-Stokes equations," SIAM J. Numer. Anal., v. 21, 1984, pp. 615-634.

7. G. Cooperman, $\alpha$-Condensing Maps and Dissipative Systems, Ph.D. Thesis, Brown University, Providence, RI, June 1978.

8. M. CROUZEIX \& V. THOMEE, "On the discretization in time of semilinear parabolic equations with nonsmooth initial data," Math. Comp., v. 49, 1987, pp. 359-377.

9. M. CROUzeIX \& V. THOMÉE, "The stability in $L_{p}$ and $W_{p}^{1}$ of the $L_{2}$-projection onto finite element function spaces," Math. Comp., v. 48, 1987, pp. 521-532.

10. H. FUJita \& A. MiZUTANI, "On the finite element method for parabolic equations. I. Approximation of holomorphic semigroups," J. Math. Soc. Japan, v. 28, 1976, pp. 749-771.

11. J. K. HALE, "Some recent results on dissipative processes," in Functional Differential Equations and Bifurcations (Ize, ed.), Lecture Notes in Math., vol. 799, Springer-Verlag, Berlin and New York, 1980, pp. 152-172.

12. J. K. HALE, "Asymptotic behavior and dynamics in infinite dimensions," in Research Notes in Math., vol. 132, Pitman, 1985, pp. 1-42.

13. J. K. Hale, J. P. LASalle, \& M. SlemRod "Theory of a general class of dissipative processes," J. Math. Anal. Appl., v. 39, 1972, pp. 177-191.

14. J. K. HALE \& O. LOPES, "Fixed point theorems and dissipative processes," J. Differential Equations, v. 13, 1973, pp. 391-402.

15. J. K. HALE, X. B. LIN \& G. RAUGEL, Upper Semicontinuity of Attractors for Approximations of Semigroups and Partial Differential Equations, LCDS report \#85-29, Brown University, Providence, RI, October 1985.

16. H. P. HELFRICH, "Fehlerabschätzungen für das Galerkinverfahren zur Lösung von Evolutionsgleichungen," Manuscripta Math., v. 13, 1974, pp. 219-235.

17. D. Henry, Geometric Theory of Semilinear Parabolic Equations, Lecture Notes in Math., vol. 840, Springer-Verlag, Berlin and New York, 1981.

18. J. G. HEYWOOD \& R. RANNACHER, "Finite element approximation of the nonstationary Navier-Stokes problem, Part II: Stability of solutions and error estimates uniform in time," SIAM J. Numer. Anal., v. 23, 1986, pp. 750-777. 
19. J. G. HEYWOOD \& R. RANNACHER, "Finite element approximation of the nonstationary Navier-Stokes problem, Part III: Smoothing property and higher order error estimates for partial discretization." (Preprint.)

20. C. Johnson, S. LARSSON, V. ThOMÉE \& L. B. WAHLBIN "Error estimates for spatially discrete approximations of semilinear parabolic equations with nonsmooth initial data," Math. Comp., v. 49, 1987, pp. 331-357.

21. T. KATO, "Fractional powers of dissipative operators II," J. Math. Soc. Japan, v. 14, 1962 , pp. 242-248.

22. S. N. S. KhALSA, "Finite element approximation of a reaction diffusion equation. Part I: Application of a topological technique to the analysis of asymptotic behavior of the semidiscrete approximation." (Preprint.)

23. O. A. LADYZhenskayA, "A dynamical system generated by the Navier-Stokes equations," Zap. Nauchn. Sem. Leningrad. Otdel. Mat. Inst. Steklov. (LOMI), v. 27, 1972, pp. 91-115.

24. O. A. LADYZhensKayA, "Dynamical system generated by the Navier-Stokes equations," Soviet Phys. Dokl., v. 17, 1973, pp. 647-649.

25. X. B. LIN \& G. RAUGEL, "Approximation of attractors of Morse-Smale systems given by parabolic equations." (In preparation.)

26. J. L. LIONS, "Espaces d'interpolation et domaines de puissances fractionnaires d'opérateurs," J. Math. Soc. Japan, v. 14, 1962, pp. 234-241.

27. P. MASSATT, "Attractivity properties of $\alpha$-contractions," J. Differential Equations, v. 48, 1983, pp. 326-333.

28. X. MORA, "Comparing the phase portrait of a nonlinear parabolic equation with that of its Galerkin approximations." (Preprint).

29. G. RAUGEL, "Hilbert space estimates in the approximation of inhomogeneous parabolic problems by single step methods," submitted to Math. Comp.

30. P. RUTKOWSKI, "Approximate solutions of eigenvalue problems with reproducing nonlinearities," Z. Angew. Math. Phys., v. 34, 1983, pp. 310-321.

31. K. SChMitT, R. C. Thompson \& W. WAlter, "Existence of solutions of a nonlinear boundary value problem via the method of lines," Nonlinear Anal., v. 2, 1978, pp. 519-535.

32. R. TÉmAM, Navier-Stokes Equations and Nonlinear Functional Analysis, CBMS-NSF, vol. 41, SIAM, Philadelphia, Pa., 1983.

33. V. Tноме́E, Galerkin Finite Element Methods for Parabolic Problems, Lecture Notes in Math., vol. 1054, Springer-Verlag, Berlin and New York, 1984.

34. V. ThOMÉE \& L. WAHLBIN, "On Galerkin methods in semilinear parabolic problems," SIAM J. Numer. Anal., v. 12, 1975, pp. 378-389. 